\title{
Hybrid Control of Induction Motors via Sampled Closed Representations
}

\author{
Bernardino Castillo-Toledo, Stefano Di Gennaro, Alexander G. Loukianov, and Jorge Rivera
}

\begin{abstract}
In this paper, a controller for induction motors is proposed. A continuous feedback is first applied to obtain a discrete-time model in closed form. Then, on the basis of these exact sampled dynamics, a discrete-time controller ensuring speed and flux modulus reference tracking is determined, making use of the sliding mode technique. The resulting controller is hence hybrid, in the sense that it contains both continuous and discretetime terms. It is shown how to implement such a hybrid controller using the so-called exponential holder, which is the only device to be implemented analogically, together with an analog integrator. Moreover, a discrete-time reduced-order observer is designed for rotor fluxes and load torque estimation. The performance of the proposed controller is finally studied by simulation and experimental tests.
\end{abstract}

Index Terms-Exponential holder, induction motors (IMs), sampled closed form, sliding mode control.

\section{INTRODUCTION}

$\mathbf{I}$ NDUCTION motors (IMs), due to the characteristics of their nonlinear dynamics, constitute an interesting test bed for the synthesis of advanced control laws. Moreover, IMs are widely used in industrial applications. Some of the reasons of their success are their simple mechanical construction, their low maintenance requirements, and the lower cost compared to other devices, such as dc or stepper motors. Starting from the pioneering work of [6], field-oriented control has become a classical technique for IM control, which involves nonlinear state transformation and feedback for the asymptotic decoupling of the rotor velocity and flux modulus [12]. More recently, various nonlinear control design approaches have been considered to improve the performance. Active research areas include input-output feedback linearization [27], passivity [19], [44], adaptive backstepping [25], [42], and sliding modes [11], [24], [39], [40], [43], [45], [48], among others.

Manuscript received July 23, 2007; revised June 23, 2008. First published July 9, 2008; current version published October 1, 2008. This work was supported in part by the Consiglio Nazionale delle Ricerche (Italy), by the Ministero degli Affari Esteri (Italy), and by the Consejo Nacional de Ciencia y Tecnología (México) under Project 46538. This paper was presented in part at the 2004 American Control Conference, Boston, MA, USA.

B. Castillo-Toledo and A. G. Loukianov are with the Research and Advanced Studies Center of the National Polytechnic Institute, 45010 Gaudalajara, Jalisco, México (e-mail: toledo@gdl.cinvestav.mx; louk@gdl.cinvestav.mx).

S. Di Gennaro is with the Department of Electrical and Information Engineering and Center of Excellence DEWS, University of L'Aquila, 67040 L'Aquila, Italy (e-mail: digennar@ing.univaq.it).

J. Rivera is with the Centro Universitario de Ciencias Exactas e Ingenierías, Departamento de Electrónica, Universidad de Guadalajara, 44430, Guadalajara, Jalisco, México (e-mail: jorge.rivera@ cucei.udg.mx).

Color versions of one or more of the figures in this paper are available online at http://ieeexplore.ieee.org.

Digital Object Identifier 10.1109/TIE.2008.928117
The implementation of control laws designed for these devices is nowadays generally made in discrete time. In fact, the recent advancements in digital microprocessor technology have rendered cheaper, simpler, and more flexible the discretetime implementation of controllers designed using continuous time techniques. The main problem here is the degradation of the expected performance, due to the fact that the design of the control law is not made using the sampled dynamics of the system. It is worth noting that the determination of an appropriate sampling period and the evaluation of its influence on the continuous controller performance are important issues, and presently, a well-recognized methodology to deal with these aspects has not been established yet.

This problem has motivated in the last decades the work of various researchers, with the aim of improving the control performance by designing the controller directly on the basis of the digital model (see [37], [38], and the references therein). Once the digital model has been obtained, various important issues regarding the controller performance can be faced, such as parameter variations, observer design, determination of the sampling period, modeling of the actuator's dynamics, etc. Clearly, the quality of the solutions given in this way depend on the accuracy of the digital model. In this regard, unfortunately, the problem of sampling continuous time systems is not trivial. In fact, in general, a sampled closed representation of the sampled dynamics does not exist; whereas for linear systems, a sampled model in closed form can be easily obtained [4], for nonlinear systems in general, the sampled data representations are given in the form of infinite series [28], [29], [33], [34], [37]. Hence, in practice, one uses truncated models of desired approximation [13], [18]. This difficulty motivates the following possible solutions:

1) the design of the control law in the continuous time setting and its implementation by means of zero-order holder;

2) the use of much simpler discretization methods, such as those due to Euler (backward or forward), Tustin, etc., and the design of the control law in the digital setting.

The first solution has the drawback of possible poor performance of the resulting sampled controller. In order to recover this performance, a multirate control approach can be pursued, see [31] and its application to synchronous motors [35], [36] or to rigid spacecraft [15]. The second solution [13] has the disadvantage that the accuracy of the resulting approximate discretetime system decreases as the sampling period increases.

An alternative to these approaches is presented in [16] and [32], where an original hybrid control methodology is 
presented. First, a continuous control puts the system in a finite discretizable form, and then, a discrete control satisfies the control objectives. An advantage of obtaining an exact discrete-time model is that the sampling period to be used in the implementation of the control law can be high, at least theoretically. Other aspects, such as actuators, data acquisition, intersampling behavior, etc., limit in practice its value. Clearly, this methodology can be applied, provided that the continuous control can be applied by means of analog devices. In this sense, the resulting controller is hybrid, because it applies a continuous control plus a digital one. It is evident that this is the critical point of this methodology. In fact, if the control can be applied only in discrete time, the continuous control has to be sampled, so that, eventually, the system is not in a closed form.

Following this idea, in this paper, we will show how to implement for IMs a continuous control which puts the system in a form so that a sampled closed representation does exist. We will show that, in this continuous control, the sampled values of the variables, necessary to implement the control, appear along with an exponential term, usually called exponential holder. The latter is the only item to be implemented via an analog device, easily realizable, together with an analog integrator. In a certain sense, the exponential holder and the analog integrator represent the "minimum requisites" that have to be implemented analogically. Once the sampled data representation in closed form of the IM dynamics has been obtained, we design a digital controller necessary to impose the control specifications, i.e., for rotor angular velocity and rotor flux square modulus tracking. A discrete-time reduced-order observer is then designed to avoid the measurement of load torque and rotor flux, the latter achievable by Hall sensors, quite imprecise and expensive to place within the motor.

Hence, analogously to the case of finite discretizability [16], [32], we need to determine first a continuous feedback in order to obtain a closed representation of the sampled dynamics. It will be shown that, in our case, such a closed form can be obtained by means of a simple control. The resulting system is then characterized by the dynamics of fluxes and currents which are linear in appropriate coordinates. Using the solutions of these dynamics, the derivation of the discrete-time equations for rotor angular position and velocity is then possible.

This paper is organized as follows. In Section II, the continuous time IM model is briefly reviewed and the exact sampled dynamics of the IM are derived. In Section III, a discrete-time sliding mode control for rotor angular velocity and rotor flux square modulus tracking is designed. To remove the hypothesis of rotor flux and load torque measurements, a reduced discretetime observer is proposed in Section IV. Moreover, the practical implementation of the proposed controller by means of an exponential holder are discussed. Section V presents some simulation and experimental results. Final comments conclude this paper.

\section{SAMPLED DYNAMICS OF IMs}

In the following, a sampled version of the dynamics of an IM will be determined. Under the assumptions of equal mutual inductance and linear magnetic circuit, the IM model in the stator reference frame $(a, b)$ is given by [27]

$$
\begin{aligned}
\dot{\phi}_{r} & =-\alpha \phi_{r}+p \omega \Im \phi_{r}+\alpha L_{m} I_{s} \\
\dot{I}_{s} & =\alpha \beta \phi_{r}-p \beta \omega \Im \phi_{r}-\gamma I_{s}+\frac{1}{L_{\sigma}} u \\
\dot{\omega} & =\mu I_{s}^{T} \Im \phi_{r}-\frac{1}{J} T \\
\dot{\theta} & =\omega
\end{aligned}
$$

where $\theta$ and $\omega$ are the rotor angular position and velocity, respectively, $T$ is a bounded load torque, $J$ is the rotor moment of inertia

$$
\phi_{r}=\left(\begin{array}{c}
\phi_{r, a} \\
\phi_{r, b}
\end{array}\right) \quad I_{s}=\left(\begin{array}{c}
i_{s, a} \\
i_{s, b}
\end{array}\right) \quad u=\left(\begin{array}{l}
u_{a} \\
u_{b}
\end{array}\right)
$$

are the rotor flux vector, the stator current vector, and the control input voltage vector, respectively, and

$$
\begin{aligned}
& \alpha=\frac{R_{r}}{L_{r}} \quad \beta=\frac{L_{m}}{L_{\sigma} L_{r}} \quad \gamma=\frac{1}{L_{\sigma}}\left(\frac{L_{m}^{2}}{L_{r}^{2}} R_{r}+R_{s}\right) \\
& L_{\sigma}=L_{s}-\frac{L_{m}^{2}}{L_{r}} \quad \mu=\frac{3}{2} \frac{p L_{m}}{J L_{r}} \quad \Im=\left(\begin{array}{cc}
0 & -1 \\
1 & 0
\end{array}\right) .
\end{aligned}
$$

Here, $L_{s}, L_{r}$, and $L_{m}$ are the stator, rotor, and mutual inductances, respectively, $R_{s}$ and $R_{r}$ are the stator and rotor resistances, and $p$ is the number of pole pairs. It is worth noting that $\Im$ represents the anticlockwise rotation operator of $\pi / 2$.

The following assumption, acceptable when $T$ varies slowly with respect to the electrical dynamics [45], will be instrumental for deriving the sampled model of the motor dynamics.

$\left(H_{1}\right)$ : The load torque $T$ can be approximated by a signal $C$ which is piecewise constant over the sampling period $\delta$.

In order to obtain sampled dynamics of the system dynamics (1) in closed form, in the spirit of [16] and [32], let us consider first the following feedback:

$$
u=u_{f}(t)+e^{p \theta \Im} v_{k}, \quad u_{f}=p L_{\sigma} \omega \Im\left(I_{s}+\beta \phi_{r}\right)
$$

with $u_{f}$ as the continuous control which makes the system finite discretizable.

Remark 1: Note that $u_{f}$ in (2) and the term $e^{p \theta \Im}$ must be implemented by analog devices, whereas the new control $v_{k}=$ $v(k \delta)=\left(\begin{array}{ll}v_{a, k} & v_{b, k}\end{array}\right)^{T}$, designed on the basis of the discretetime representation of the system derived in the following, can be implemented by a digital computer and a zero-order holder. In Section IV, it will be explained how to determine $u_{f}$ from the sampled measurements and using analog devices given by an integrator and a so-called exponential holder [8], [9]. The resulting controller will be hybrid, namely it will contain discrete and continuous time terms.

With the continuous feedback $(2)$ and under $\left(H_{1}\right)$, the following controlled dynamics are obtained:

$$
\begin{aligned}
\dot{\phi}_{r} & =-\alpha \phi_{r}+p \omega \Im \phi_{r}+\alpha L_{m} I_{s} \\
\dot{I}_{s} & =\alpha \beta \phi_{r}-\gamma I_{s}+p \omega \Im I_{s}+\frac{1}{L_{\sigma}} e^{p \theta \Im} v_{k} \\
\dot{\omega} & =\mu I_{s}^{T} \Im \phi_{r}-\frac{1}{J} C \\
\dot{\theta} & =\omega .
\end{aligned}
$$


As common in the IM's literature [27], we consider hereinafter a change of coordinates in an appropriate rotating frame [23]. In particular, we consider a transformation which puts in light that the flux and current dynamics are linear, with the current dynamics linear, thanks to the feedback (2). Hence, we consider the globally defined change of coordinates

$$
\left(\begin{array}{c}
\Phi \\
I \\
\omega \\
\theta
\end{array}\right)=\left(\begin{array}{c}
e^{-p \theta \Im} \phi_{r} \\
e^{-p \theta \Im} I_{s} \\
\omega \\
\theta
\end{array}\right), \quad e^{-p \theta \Im}=\left(\begin{array}{cc}
\cos p \theta & \sin p \theta \\
-\sin p \theta & \cos p \theta
\end{array}\right)
$$

with $e^{-p \theta \Im}$ as an orthogonal matrix. The transformed variables $\Phi$ and $I$ in (4) are the rotor flux and the stator current rotated according to the electrical rotor angular position $p \theta$, respectively. Obviously, (4) preserves the vector norms. Note also that the complex notation $e^{-j p \theta}\left(V_{a}+j V_{b}\right)$ could be equivalently used for the terms $e^{-p \theta \Im} V, V=\phi_{r}$, and $I_{s}$ in (4).

In the new variables [(4)] and with the input $v_{k}$, the dynamics [(3)] are expressed as follows:

$$
\begin{aligned}
\dot{\Phi} & =-\alpha \Phi+\alpha L_{m} I \\
\dot{I} & =\alpha \beta \Phi-\gamma I+\frac{1}{L_{\sigma}} v_{k} \\
\dot{\omega} & =\mu I^{T} \Im \Phi-\frac{1}{J} C \\
\dot{\theta} & =\omega
\end{aligned}
$$

because $d e^{-p \theta \Im} / d t=-p \omega \Im e^{-p \theta \Im}$ and $e^{-p \theta \Im} \Im=\Im e^{-p \theta \Im}$. Note that the equations in (5) are nonlinear, but the sampled closed form is now easily obtained noting that the dynamics for $\Phi$ and $I$ are linear, and the control $v_{k}$ will be designed to be constant over the sampling period $\delta$. Denoting $\Phi_{k}=\Phi(k \delta)$, $I_{k}=I(k \delta), \omega_{k}=\omega(k \delta)$, and $C_{k}=C(k \delta)$ and considering as outputs to be controlled the rotor angular velocity and the rotor flux square modulus, some calculations given in Appendix I yield the closed representation for the sampled dynamics of system (5)

$$
\begin{aligned}
\Phi_{k+1} & =a_{11} \Phi_{k}+a_{12} I_{k}+b_{1} v_{k} \\
I_{k+1} & =a_{21} \Phi_{k}+a_{22} I_{k}+b_{2} v_{k} \\
\omega_{k+1} & =\omega_{k}+\eta_{1} I_{k}^{T} \Im \Phi_{k}+\left(\eta_{2} \Phi_{k}+\eta_{3} I_{k}\right)^{T} \Im v_{k}-\frac{C_{k}}{J} \delta \\
\theta_{k+1} & =\theta_{k}+\omega_{k} \delta+\kappa_{1} I_{k}^{T} \Im \Phi_{k}+\left(\kappa_{2} \Phi_{k}+\kappa_{3} I_{k}\right)^{T} \Im v_{k}-\frac{C_{k}}{J} \frac{\delta^{2}}{2}
\end{aligned}
$$

where $a_{11}, a_{12}, a_{21}, a_{22}, b_{1}, b_{2}, \eta_{i}$, and $\kappa_{i}, i=1,2,3$, are constants. Note that the matrix

$$
A_{d}=\left(\begin{array}{cc}
a_{11} \mathbb{I}_{2 \times 2} & a_{12} \mathbb{I}_{2 \times 2} \\
a_{21} \mathbb{I}_{2 \times 2} & a_{22} \mathbb{I}_{2 \times 2}
\end{array}\right)=A \otimes \mathbb{I}_{2 \times 2}
$$

with $\mathbb{I}_{2 \times 2}$ as the identity matrix $2 \times 2$, and $\otimes$, the Kronecker product, is Schur because $A$ is Schur for every choice of the sampling period $\delta$ (see Appendix I).

The control problem is to force the rotor angular velocity $\omega_{k}$ and the rotor flux modulus $\Phi_{k}^{T} \Phi_{k}$ to track some desired references $\omega_{r, k}$ and $\Phi_{r, k}$, ensuring at the same time disturbance rejection. We assume that these references are bounded with bounded increments, where

$$
\begin{aligned}
\left|\Delta \omega_{r, k}\right| & =\left|\omega_{r, k+1}-\omega_{r, k}\right| \leq \Delta \omega_{r, \max } \\
\left|\Delta \Phi_{k}\right| & =\left|\Phi_{r, k+1}-\Phi_{r, k}\right| \leq \Delta \Phi_{\max } .
\end{aligned}
$$

Moreover, also the load torque $C_{k}$ is bounded by a value $C_{\max }$. Furthermore, we assume that $C_{k}$ has bounded increments. Finally, we need to ensure that this increment vanishes when $\delta$ is zero. This can be formalized with the following assumption.

$\left(H_{2}\right)$ : The piecewise load torque $C_{k}$ is such that $C_{k+1}=$ $C_{k}+\Delta C(k, \delta)$ with, for each integer $\bar{k}$

$$
|\Delta C(\bar{k}, \delta)| \leq \Delta C_{\max } \Delta(\delta), \quad \lim _{\delta \rightarrow 0} \Delta(\delta)=0 .
$$

The control problem will be solved in the next section by means of a discrete-time sliding mode control.

Remark 2: As last observation, it is interesting to compare $u_{f}$ in (2) with the well-known field-oriented control (FOC) [12]. The FOC can be determined considering the $(d, q)$ model

$$
\begin{aligned}
\dot{\phi}_{r, d q} & =-\alpha \phi_{r, d q}+p \omega \Im \phi_{r, d q}+\alpha L_{m} I_{s, d q}-\dot{\epsilon}_{0} \Im \phi_{r, d q} \\
& =\left(\begin{array}{c}
-\alpha \phi_{r, d}+\alpha L_{m} i_{s, d} \\
0
\end{array}\right) \\
\dot{I}_{s, d q} & =\alpha \beta \phi_{r, d q}-p \beta \omega \Im \phi_{r, d q}-\gamma I_{s, d q}+\frac{1}{L_{\sigma}} u_{d q}-\dot{\epsilon}_{0} \Im I_{s, d q} \\
& =\left(\begin{array}{c}
\alpha \beta \phi_{r, d}-\gamma i_{s, d}+\dot{\epsilon}_{0} i_{s, q}+\frac{1}{L_{\sigma}} u_{d} \\
-p \beta \omega \phi_{r, d}-\gamma i_{s, q}-\dot{\epsilon}_{0} i_{s, d}+\frac{1}{L_{\sigma}} u_{q}
\end{array}\right) \\
\dot{\omega} & =\mu I_{s, d q}^{T} \Im \phi_{r, d q}-\frac{1}{J} T=\mu \phi_{r, d} i_{s, q}-\frac{1}{J} T \\
\dot{\theta} & =\omega
\end{aligned}
$$

obtained from (1) considering

$$
\begin{aligned}
& I_{d q}=\left(\begin{array}{c}
i_{s, d} \\
i_{s, q}
\end{array}\right)=e^{-\epsilon_{0} \Im} I_{s} \quad \phi_{r, d q}=\left(\begin{array}{c}
\phi_{r, d} \\
0
\end{array}\right)=e^{-\epsilon_{0} \Im} \phi_{r} \\
& u_{d q}=\left(\begin{array}{c}
u_{d} \\
u_{q}
\end{array}\right)=e^{-\epsilon_{0} \Im} u \quad \dot{\epsilon}_{0}=p \omega+\frac{\alpha L_{m}}{\phi_{r, d}} i_{s, q} .
\end{aligned}
$$

It is not surprising that (9) resembles (4), because the idea behind the FOC is to have linear flux and current dynamics, as in the finite discretization. In both cases, the first one determines a transformation which renders linear the flux dynamics. Then, one looks for a control law which cancels the nonlinearities in the current dynamics. Indeed, the transformation (9) is different from (4), and the resulting models (5) and (8) are different in the current dynamics. This leads to different control expressions. In fact, the FOC is given by [12]

$$
\begin{aligned}
u_{d q} & =L_{\sigma}\left(-\alpha \beta \phi_{r, d q}+p \beta \omega \Im \phi_{r, d q}+\dot{\epsilon}_{0} \Im I_{s, d q}+v_{d q}\right) \\
& =L_{\sigma}\left(\begin{array}{c}
-\alpha \beta \phi_{r, d}-\dot{\epsilon}_{0} i_{s, q}-\frac{\alpha L_{m}}{\phi_{r, d}} i_{s, q}^{2}+v_{d} \\
p \beta \omega \phi_{r, d}+\dot{\epsilon}_{0} i_{s, d}+\frac{\alpha L_{m}}{\phi_{r, d}} i_{s, d} i_{s, q}+v_{q}
\end{array}\right)
\end{aligned}
$$


where $v_{d q}=\left(\begin{array}{ll}v_{d} & v_{q}\end{array}\right)^{T}$, with

$$
\begin{gathered}
v_{d}=-k_{d, 1}\left(\phi_{r, d}-\phi_{\mathrm{ref}}\right)-k_{i, 1} \int_{0}^{t}\left(\phi_{r, d}(\tau)-\phi_{\mathrm{ref}}(\tau)\right) d \tau \\
v_{q}=-k_{d, 2}\left(\omega_{r, d}-\omega_{\mathrm{ref}}\right)-k_{i, 2} \int_{0}^{t}\left(\omega_{r, d}(\tau)-\omega_{\mathrm{ref}}(\tau)\right) d \tau
\end{gathered}
$$

and $\phi_{\text {ref }}$ and $\omega_{\text {ref }}$ as the flux and angular velocity references, respectively, and $k_{d, 1}, k_{d, 2}, k_{i, 1}, k_{i, 2}>0$. One hence obtains the following closed-loop dynamics:

$$
\begin{aligned}
\dot{\phi}_{r, d q} & =-\alpha \phi_{r, d q}+p \omega \Im \phi_{r, d q}+\alpha L_{m} I_{s, d q}-\dot{\epsilon}_{0} \Im \phi_{r, d q} \\
& =\left(\begin{array}{c}
-\alpha \phi_{r, d}+\alpha L_{m} i_{s, d} \\
0
\end{array}\right) \\
\dot{I}_{s, d q} & =-\gamma I_{s, d q}+v_{d q} \\
\dot{\omega} & =\mu I_{s, d q}^{T} \Im \phi_{r, d q}-\frac{1}{J} T=\mu \phi_{r, d} i_{s, q}-\frac{1}{J} T \\
\dot{\theta} & =\omega .
\end{aligned}
$$

From (10), it is clear that FOC imposes the feedback linearization of (8) [22]. Hence, the FOC eventually obtains a linear model for the flux and current dynamics, as $u_{f}$ in (2). In this sense, the FOC can be applied to obtain a finite discretizable system as well, namely, from (11), one could obtain an exact closed form for the sampled dynamics. Indeed, it easy to see that $u_{f}$ is more general than FOC, because it cancels just the terms which do not allow the finite discretizability. In fact, in the $(d, q)$ coordinates $u_{f}$ can be written as

$$
u_{f, d q}=e^{-\epsilon_{0} \Im} u_{f}=L_{\sigma}\left(p \beta \omega \Im \phi_{r, d q}+p \omega \Im I_{s, d q}\right)
$$

and it is readily checked that

$$
u_{d q}=u_{f, d q}+L_{\sigma}\left(-\alpha \beta \phi_{r, d q}+\frac{\alpha L_{m}}{\phi_{r, d}} i_{s, q} \Im I_{s, d q}+v_{d q}\right)
$$

and it is clear that the implementation of the FOC would imply also the implementation of further terms.

\section{Discrete-Time Control of IMs}

The discrete-time control $v_{k}$ in (2) is designed hereinafter, in order to solve the posed control problem, following the sliding-mode approach. On this ground, we first derive the expression of the tracking error dynamics $e_{1, k}=\omega_{k}-\omega_{r, k}$, $e_{2, k}=\Phi_{k}^{T} \Phi_{k}-\Phi_{r, k}$, which are the new outputs which we want to force to zero. Using (6), the error dynamics can be written as follows:

$$
\begin{aligned}
e_{1, k+1}= & \omega_{k}+\eta_{1} I_{k}^{T} \Im \Phi_{k}+\lambda_{1, k}^{T} v_{k}-\frac{\delta}{J} C_{k}-\omega_{r, k+1} \\
e_{2, k+1}= & a_{11}^{2} \Phi_{k}^{T} \Phi_{k}+2 a_{11} a_{12} \Phi_{k}^{T} I_{k}+a_{12}^{2} I_{k}^{T} I_{k} \\
& +\lambda_{2, k}^{T} v_{k}+b_{1}^{2} v_{k}^{T} v_{k}-\Phi_{r, k+1}
\end{aligned}
$$

where

$$
\lambda_{1, k}=-\Im\left(\eta_{2} \Phi_{k}+\eta_{3} I_{k}\right) \quad \lambda_{2, k}=2 b_{1}\left(a_{11} \Phi_{k}+a_{12} I_{k}\right) .
$$

It is interesting to note that $\lambda_{1, k}$ represents the vector $\eta_{2} \Phi_{k}+$ $\eta_{3} I_{k}$ rotated $\pi / 2$ clockwise.

Notice that the system (12) depends on quadratic terms of the control signal $v_{k}$ because $b_{1}>0$ for all sampling time $\delta>0$. This complicates the control design procedure. In order to simplify the design of a control law, one considers as new control

$$
\mathcal{V}_{k}=\left(\begin{array}{l}
\mathcal{V}_{a, k} \\
\mathcal{V}_{b, k}
\end{array}\right)=\mathcal{T}_{k} v_{k}, \quad \mathcal{T}_{k}=\left(\begin{array}{c}
\lambda_{1, k}^{T} \\
\lambda_{2, k}^{T}
\end{array}\right)
$$

$v_{k}=\mathcal{T}_{k}^{-1} \mathcal{V}_{k}$. It can be verified that the transformation $\mathcal{T}_{k}$ is invertible for all $k$, with

$$
\operatorname{det} \mathcal{T}_{k}=d_{k}=\lambda_{2, k}^{T} \Im \lambda_{1, k}=2 b_{1}\left(\begin{array}{c}
\Phi_{k} \\
I_{k}
\end{array}\right)^{T} R\left(\begin{array}{c}
\Phi_{k} \\
I_{k}
\end{array}\right) \neq 0
$$

and

$$
\mathcal{R}=\left(\begin{array}{cc}
a_{11} \eta_{2} \mathbb{I}_{2 \times 2} & \frac{1}{2}\left(a_{12} \eta_{2}+a_{11} \eta_{3}\right) \mathbb{I}_{2 \times 2} \\
\frac{1}{2}\left(a_{12} \eta_{2}+a_{11} \eta_{3}\right) \mathbb{I}_{2 \times 2} & a_{12} \eta_{3} \mathbb{I}_{2 \times 2}
\end{array}\right)
$$

nonsingular for all $\delta$ (see Appendix I). Note that

$$
\begin{aligned}
& \mathcal{T}_{k}^{-1}=\frac{1}{d_{k}} \Im\left(\begin{array}{ll}
-\lambda_{2, k} & \lambda_{1, k}
\end{array}\right) \\
& \left(\mathcal{T}_{k}^{-1}\right)^{T}\left(\mathcal{T}_{k}^{-1}\right)=\frac{1}{d_{k}^{2}}\left(\begin{array}{cc}
\lambda_{2, k}^{T} \lambda_{2, k} & -\lambda_{1, k}^{T} \lambda_{2, k} \\
-\lambda_{1, k}^{T} \lambda_{2, k} & \lambda_{1, k}^{T} \lambda_{1, k}
\end{array}\right) .
\end{aligned}
$$

With such a control transformation, $e_{1, k+1}$ and $e_{2, k+1}$ depend only on $\mathcal{V}_{a, k}$ and $\mathcal{V}_{b, k}$, respectively, and (12) becomes

$$
\begin{aligned}
& e_{1, k+1}=\omega_{k}+\eta_{1} I_{k}^{T} \Im \Phi_{k}-\frac{\delta}{J} C_{k}-\omega_{r, k+1}+\mathcal{V}_{a, k} \\
& e_{2, k+1}=a_{k} \mathcal{V}_{b, k}^{2}+b_{k} \mathcal{V}_{b, k}+c_{k}
\end{aligned}
$$

where

$$
\begin{aligned}
a_{k}= & \frac{b_{1}^{2}}{d_{k}^{2}} \lambda_{1, k}^{T} \lambda_{1, k} \geq 0 \quad b_{k}=1-\frac{2 b_{1}^{2}}{d_{k}^{2}} \lambda_{1, k}^{T} \lambda_{2, k} \mathcal{V}_{a, k} \\
c_{k}= & \frac{1}{4 b_{1}^{2}} \lambda_{2, k}^{T} \lambda_{2, k}+\frac{b_{1}^{2}}{d_{k}^{2}} \lambda_{2, k}^{T} \lambda_{2, k} \mathcal{V}_{a, k}^{2}-\Phi_{r, k+1} \\
= & \left(\frac{1}{4 b_{1}^{2}}+\frac{b_{1}^{2}}{d_{k}^{2}} \mathcal{V}_{a, k}^{2}\right) \lambda_{2, k}^{T} \lambda_{2, k}-\Phi_{r, k+1} \\
= & \left(\begin{array}{c}
\Phi_{k} \\
I_{k}
\end{array}\right)^{T}\left(\begin{array}{cc}
a_{11}^{2} & a_{11} a_{12} \\
a_{11} a_{12} & a_{12}^{2}
\end{array}\right)\left(\begin{array}{c}
\Phi_{k} \\
I_{k}
\end{array}\right) \\
& +\frac{b_{1}^{2}}{d_{k}^{2}} \lambda_{2, k}^{T} \lambda_{2, k} \mathcal{V}_{a, k}^{2}-\Phi_{r, k+1} .
\end{aligned}
$$

It is worth noting that $a_{k}$ is zero when $\Phi_{k}=-\eta_{3} I_{k} / \eta_{2}$.

A discrete-time sliding-mode control will be designed hereinafter to force to zero the error dynamics. A natural choice as sliding functions are the errors $e_{1, k}$ and $e_{2, k}$.

Because the control objective is to design a controller for angular velocity tracking and disturbance rejection, considering $e_{1, k}$ as sliding surface, one can impose that this tracking error be zeroed in one step, namely, $e_{1, k+1}=0$. Considering the first of (16), this is achieved considering

$$
\mathcal{V}_{a, k}=-\omega_{k}-\eta_{1} I_{k}^{T} \Im \Phi_{k}+\frac{\delta}{J} C_{k}+\omega_{r, k+1} .
$$


Usually, such a control is called equivalent control and is such that the sliding manifold $e_{1, k}=0$ is rendered invariant.

Analogously, we determine $\mathcal{V}_{b, k}$, imposing that the tracking error $e_{2, k}$ is zeroed in one or more steps or tends asymptotically to zero. The determination of the equivalent control follows from simple considerations reported hereinafter.

1) If $a_{k}>0$ and $\Delta_{k}=b_{k}^{2}-4 a_{k} c_{k} \geq 0$, considering the second of (16), one imposes $a_{k} \mathcal{V}_{b, k}^{2}+b_{k} \mathcal{V}_{b, k}+c_{k}=0$. Hence, the control

$$
\mathcal{V}_{b, k}=-\frac{b_{k}}{2 a_{k}} \pm \frac{\sqrt{\Delta_{k}}}{2 a_{k}}
$$

straightforwardly imposes $e_{2, k+1}=0$, i.e., ensures that $e_{2, k}$ is zeroed in one step.

2) If $a_{k}>0$ and $\Delta_{k}<0$, then $e_{2, k}$ cannot be zeroed in one step. In this case, we can only determine $\mathcal{V}_{b, k}$ so that $e_{2, k}$ decreases at each time step. This can be achieved as follows. For a certain $r>0$, representing the duration of the decrease required on $e_{2, k}$, one iteratively imposes

$e_{2, k+j+1}=a_{k+j} \mathcal{V}_{b, k+j}^{2}+b_{k+j} \mathcal{V}_{b, k+j}+c_{k+j}=p_{j}$

where $j=0, \ldots, r$, with $p_{j}$ chosen so that $\left|e_{2, k+j+1}\right|<$ $\left|e_{2, k+j}\right|$ and such that the new discriminant

$$
\bar{\Delta}_{k, j}=b_{k+j}^{2}-4 a_{k+j}\left(c_{k+j}-p_{j}\right)
$$

is equal to a fixed minimum value $\Delta_{\min , j} \geq 0$. Hence, $p_{j}=c_{k+j}+\left(\Delta_{\min , j}-b_{k+j}^{2}\right) /\left(4 a_{k+j}\right)$. Therefore, one easily works out the values of the control $\mathcal{V}_{\beta}$, ensuring this decrease

$$
\mathcal{V}_{b, k+j}=\frac{-b_{k+j} \pm \sqrt{\Delta_{\min , j}}}{2 a_{k+j}}, \quad j=0, \ldots, r .
$$

With this choice, $e_{2, k}$ tends asymptotically to zero. Clearly, the fastest decrease of $e_{2, k}$ is obtained for $\Delta_{\min , j}=0$, i.e., with $\mathcal{V}_{b, k+j}=b_{k+j} /\left(2 a_{k+j}\right), j=$ $0, \ldots, r$, namely, imposing $\mathcal{V}_{b, k}=b_{k} /\left(2 a_{k}\right)$ when $a_{k}>$ 0 and $\Delta_{k}<0$. Clearly, if after $r$ steps, $\Delta_{k}$ becomes nonnegative, $\mathcal{V}_{b, k}$ will be chosen as in point 1) [or 3)], therefore obtaining zero error in $r+1$ steps.

3) If $a_{k}=0$, then it is possible to impose straightforwardly $e_{2, k+1}=0$ in one step with the control $\mathcal{V}_{b, k}=-c_{k} / b_{k}$.

We can resume this discussion saying that, with the following equivalent control:

$$
\mathcal{V}_{b, k}= \begin{cases}-\frac{b_{k}}{2 a_{k}} \pm \frac{\sqrt{\Delta_{k}}}{2 a_{k}} & \text { if } a_{k}>0 \text { and } \Delta_{k} \geq 0 \\ -\frac{b_{k}}{2 a_{k}} & \text { if } a_{k}>0 \text { and } \Delta_{k}<0 \\ -\frac{c_{k}}{b_{k}} & \text { if } a_{k}=0\end{cases}
$$

with $\Delta_{k}=b_{k}^{2}-4 a_{k} c_{k}$, the flux tracking error is zeroed in one or more steps or tends asymptotically to zero.

We have shown that $e_{1, k}$ and $e_{2, k}$ are forced to zero asymptotically. One should ensure that $I_{k}$ does not diverge to infinity. This aspect is correlated with the stability of what are called the zero dynamics which, roughly speaking, are those dynamics obtained when the output of the system is zero (see [22] for the continuous time setting and [30] for the discrete-time case). We do not carry out a formal study of this aspect (but, in Appendix III, we give some more details), and we just mention that $I_{k}$ remains bounded (this can be also seen from the experimental results given in the following), whereas the flux angular position $\theta_{\Phi, k}$ and the rotor angular position $\theta_{k}$ in practice take value in $[-\pi, \pi)$. This prevents the control $v_{k}$ from saturating, and the suggested scheme can be applied to solve the posed control problem.

\section{DisCRETE-Time CONTROL FROM SAMPLED MEASUREMENTS}

In this section, we consider and fix the drawbacks of the proposed controls (2) and (14). The first problem is the measurability of the rotor flux and the load torque. This problem is solved by a simple discrete-time reduced-order observer, as shown in Section IV-A. The second problem concerns how the continuous feedback (2) is implemented, i.e., the availability of the signals $\theta(t), I_{s}(t)$, and $\phi_{r}(t)$. This aspect is considered in Section IV-B.

\section{A. Flux and Load Torque Observers}

In practical cases, the rotor flux and load torque are not measured, and this is a crucial aspect in the real implementations. The literature presents various works on this subject, in the case of continuous time, see [1], [3], [5], [14], [21], [24], [39], [41], and [46] among the others. Less numerous are the results in the discrete-time setting [2], [7], [10]. In the following, we derive flux and torque discrete-time observers for the discrete model (6). The flux observer is proposed to have the following (quite obvious) form:

$$
\hat{\Phi}_{k+1}=a_{11} \hat{\Phi}_{k}+a_{12} I_{k}+b_{1} v_{k} .
$$

Then, the dynamical error equation becomes

$$
e_{\Phi, k+1}=a_{11} e_{\Phi, k} \quad e_{\Phi, k}=\Phi_{k}-\hat{\Phi}_{k} .
$$

It can be verified that $\left|a_{11}\right|<1$ (see expression in Appendix I). Hence, $\hat{\Phi}_{k}$ asymptotically converges to $\Phi_{k}$.

As far as the load torque estimation is concerned, we have assumed that $\left(H_{2}\right)$ holds. Then, the following observer can be used:

$$
\begin{aligned}
\hat{\omega}_{k+1}= & \omega_{k}+\eta_{1} I_{k}^{T} \Im \hat{\Phi}_{k}+\left(\eta_{2} \hat{\Phi}_{k}^{T}+\eta_{3} I_{k}^{T}\right) \Im v_{k} \\
& -\frac{\delta}{J} \hat{C}_{k}+l_{1}\left(\omega_{k}-\hat{\omega}_{k}\right) \\
\hat{C}_{k+1}= & \hat{C}_{k}+l_{2}\left(\omega_{k}-\hat{\omega}_{k}\right) .
\end{aligned}
$$

Note that the estimation $\hat{\omega}_{k}$ is necessary only to determine $\hat{C}_{k}$. Setting $e_{\omega, k}=\omega_{k}-\hat{\omega}_{k}$ and $e_{C, k}=C_{k}-\hat{C}_{k}$ as rotor angular velocity and load torque estimate errors, respectively, the dynamical error equations become

$$
\begin{aligned}
\left(\begin{array}{l}
e_{\omega, k+1} \\
e_{C, k+1}
\end{array}\right)= & \left(\begin{array}{cc}
-l_{1} & -\frac{\delta}{J} \\
-l_{2} & 1
\end{array}\right)\left(\begin{array}{l}
e_{\omega, k} \\
e_{C, k}
\end{array}\right) \\
& +\left(\begin{array}{c}
\eta_{1} I_{k}^{T}-\eta_{2} v_{k}^{T} \\
0
\end{array}\right) \Im e_{\Phi, k} \\
& +\left(\begin{array}{l}
0 \\
1
\end{array}\right) \Delta C(k, \delta) .
\end{aligned}
$$


Note that $e_{\Phi, k}$ tends asymptotically to zero and $\eta_{1} I_{k}^{T}$ and $\eta_{2} v_{k}^{T}$ are bounded terms. Due to the presence of the term $\Delta C(k, \delta)$, which acts as a perturbation, choosing $l_{1}$ and $l_{2}$ such that the state matrix in (21) is Schur, $\hat{\omega}_{k}$ and $\hat{C}_{k}$ converge asymptotically to $\omega_{k}$ and $C_{k}$ in a practical sense [17], namely, the trajectory of the estimation error belongs to a neighborhood of radius $r$ of the origin. Note that, on the basis of the assumption $\left(H_{2}\right)$, the perturbation term $\Delta C(k, \delta)$ can be made small by reducing the sampling period $\delta$, namely

$$
|\Delta C(k, \delta)| \leq \Delta C_{\max } \Delta(\delta) \leq \Delta C_{\max } \Delta\left(\delta_{0}\right)
$$

for $\delta \leq \delta_{0}$, with $\delta_{0}$ sufficiently small. In this way, one obtains a smaller value for the radius $r$ and, hence, a better observer performance.

\section{B. Implementation of (2)}

The main issue concerning the implementation of the control law designed here is the realization of the continuous part of control (2), where the stator current $I_{s}(t)$, the rotor flux $\phi_{r}(t)=$ $e^{p \theta(t) \Im} \Phi(t)$, and the rotor angular position and velocity $\theta(t)$ and $\omega(t)$, respectively, are required. On this ground, we show here that this continuous control can be written as a combination of the sampled values $\theta_{k}, \omega_{k}$, and $\hat{\Phi}_{k}$. The coefficients of this combination depend on a time varying term, usually called exponential holder. This exponential holder is the only term to be implemented via an analog device, together with an analog integrator.

The stator current $I_{s}$ can be measured by analog devices, whereas the rotor angular position and velocity $\theta$ and $\omega$, respectively, are commonly measured by a digital device, such as an encoder, meaning that their values $\theta_{k}$ and $\omega_{k}$ are available at sampling instants. Furthermore, $\phi_{r}$ is estimated by the discretetime observers (19) and (20), and only the values $\hat{\phi}_{r, k}=$ $e^{p \theta_{k} \Im} \hat{\Phi}_{k}$ are available at sampling instants.

Therefore, we need to reproduce the continuous time signals $\theta(t), \omega(t)$, and $\hat{\phi}_{r}(t)$ from the knowledge of the values $\theta_{k}, \omega_{k}$, and $\hat{\Phi}_{k}$. This can be done as follows. Referring to Appendix I and considering (24) and (25), setting $\tau=t-k \delta, \tau \in[0, \delta)$, and considering $\hat{\Phi}_{k}$ in the place of $\Phi_{k}$, for $\hat{\Phi}(t)$ and $\omega(t)$, one obtains the following expressions:

$$
\begin{aligned}
\hat{\Phi}(t)= & \hat{\Phi}(\tau+k \delta)=\varphi_{1}(\tau) \hat{\Phi}_{k}+\varphi_{2}(\tau) I_{k}+\zeta_{1}(\tau) v_{k} \\
\omega(t)= & \omega(\tau+k \delta) \\
= & \omega_{k}+\nu_{1}(\tau) I_{k}^{T} \Im \hat{\Phi}_{k} \\
& +\nu_{2}(\tau) \hat{\Phi}_{k}^{T} \Im v_{k}+\nu_{3}(\tau) I_{k}^{T} \Im v_{k}-\frac{C_{k}}{J} \tau .
\end{aligned}
$$

Note that the expressions of $\varphi_{1}(\tau), \varphi_{2}(\tau), \zeta_{1}(\tau)$, and $\nu_{i}(\tau)$, $i=1,2,3$ contain terms of the form $e^{\varsigma \tau}, \varsigma= \pm \rho$ or $\varsigma= \pm \omega_{0}$, see Appendix I. In fact, the hyperbolic trigonometric functions can be represented with exponential terms. The term $e^{\varsigma \tau}$ is usually called exponential holder and is physically realizable by an analog device [8], [9]. In particular, in [9], an electronic realization of such device is proposed and a circuit realization is given for sinusoidal signals. In some sense, the exponential holder represents the "minimum requisite" that has to be implemented analogically to reproduce $\hat{\Phi}(t)$ and $\omega(t)$.
Therefore, (22) allows us to reconstruct the values of $\hat{\Phi}(t)$ and $\omega(t)$, for all $t$. Once $\omega(t)$ has been reconstructed, it can be integrated in order to obtain $\theta(t)$ by means of an analog integrator device.

\section{Simulation And ExPERImental Results}

In this section, we first compare the proposed control scheme with different control laws, by means of simulations, and then, we describe the experimental results.

\section{A. Simulation Results}

We considered an IM with the following plate data: three phase, two pole, $0.14 \mathrm{KW}, 0.74 \mathrm{pf}, 1600 \mathrm{r} / \mathrm{min}, 220 \mathrm{~V}, 1.3 \mathrm{~A}$, and with $R_{r}=10.1, R_{s}=14 \Omega, L_{s}=400, L_{r}=412.8, L_{m}=$ $377 \mathrm{mH}$, and $J=0.01 \mathrm{Kg} \mathrm{m}^{2}$.

Hence, $\alpha=24.467 \Omega / \mathrm{H}, \beta=16.3981 / \mathrm{H}, \gamma=402.62 \Omega / \mathrm{H}$, $\mu=2.73981 /\left(\mathrm{Kg} \mathrm{m}^{2}\right)$, and $L_{\sigma}=5.5695 \times 10^{-2} \mathrm{H}$. Moreover, $\omega_{0}=198.6 \Omega / \mathrm{H}$ and $\rho=213.5 \Omega / \mathrm{H}$, where $\Omega / \mathrm{H}=\mathrm{Hz}$. The sampling period is $\delta=230 \mu$ s, which is a typical value in applications, see for instance [5], and which is in accordance with the value used in Section V-B. It is worth noting that the choice of the sampling period is an important aspect in the digital implementation of control laws, and one popular way to check the influence of the sampling period $\delta$ on the controller performance is by simulation. In Section I, we have already noted that an advantage of obtaining an exact discretetime model is that the sampling period can be theoretically high, although other aspects limit in practice its value.

The gain control parameters have been chosen equal to $l_{1}=0.0824$ and $l_{2}=-0.8244$. With these values, it is possible to check that matrix (7) is Schur. A load torque $T$ of $0.2 \mathrm{Nm}$, with a variation to $1 \mathrm{Nm}$ at $t=4.6 \mathrm{~s}$, has been considered in the simulations.

In order to compare different control solutions, we have considered four control schemes:

a) the sampling of the classical FOC, see (10), with the state observer proposed in [47];

b) digital control and observer determined on the basis of the Euler approximation [26]

$$
\begin{aligned}
\phi_{r, k+1} & =\phi_{r, k}+\delta\left(-\Psi_{k} \phi_{r, k}+\alpha L_{m} I_{s, k}\right) \\
I_{s, k+1} & =I_{s, k}+\delta\left(\beta \Psi_{k} \phi_{r, k}-\gamma I_{s, k}+\frac{1}{L_{\sigma}} u_{k}\right) \\
\omega_{k+1} & =\omega_{k}+\delta\left(\mu I_{s, k}^{T} \Im \phi_{r, k}-\frac{1}{J} T_{k}\right) \\
\theta_{k+1} & =\theta_{k}+\delta \omega_{k}
\end{aligned}
$$

of the dynamics (1), with $\Psi_{k}=\alpha I_{d}-p \omega_{k} \Im$ and $I_{d}$ as the identity matrix;

c) control law given by (14), (19), and (20) and the sampling of $u_{f}$ in (2), namely

$$
u_{k}=p L_{\sigma} \omega_{k} \Im\left(I_{s, k}+\beta \hat{\phi}_{r, k}\right)+e^{p \theta_{k} \Im} v_{k}
$$

where

$$
\left(\begin{array}{c}
\hat{\phi}_{r, k} \\
I_{s, k}
\end{array}\right)=\left(\begin{array}{c}
e^{p \theta_{k} \Im} \hat{\Phi}_{k} \\
e^{p \theta_{k} \Im} I_{k}
\end{array}\right) .
$$



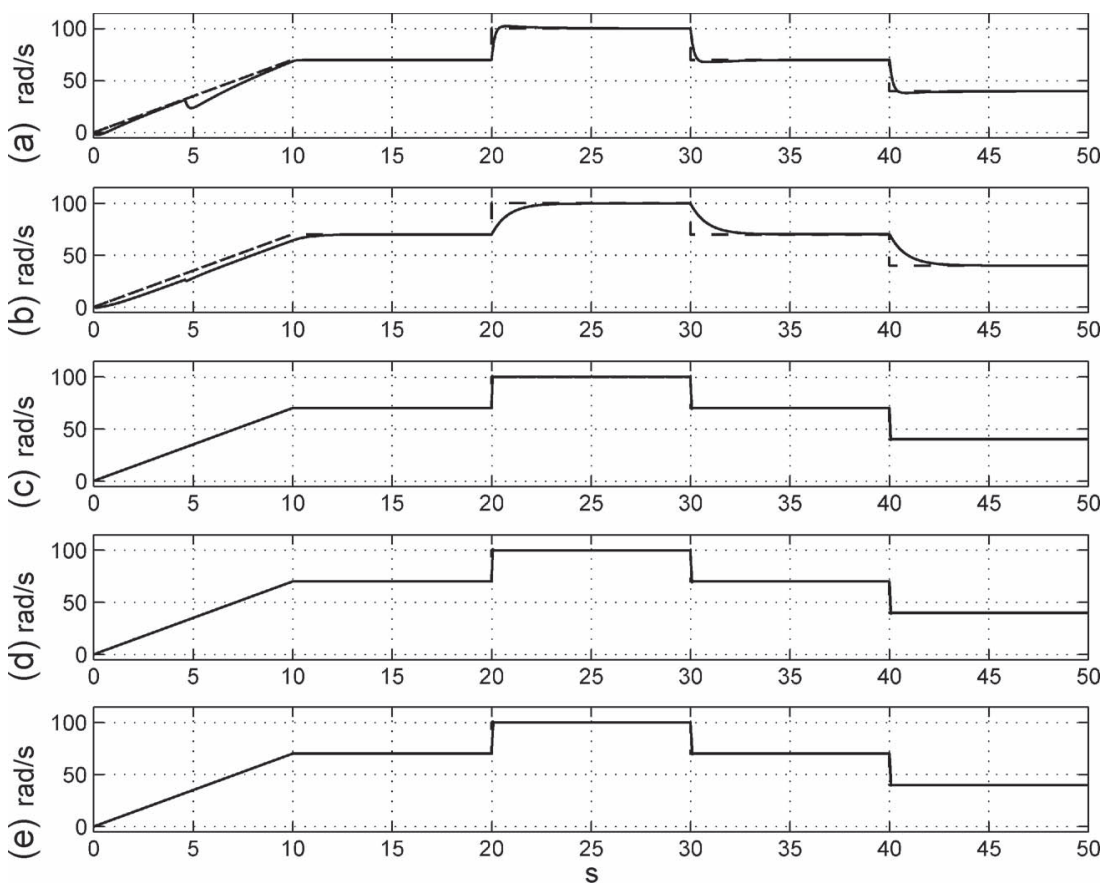

Fig. 1. (Solid) Rotor angular velocity $\omega_{k}$ and (dashed) reference $\omega_{r, k}$. (a) FOC with observer. (b) Digital control and observer (Euler approximation). (c) Control laws (14), (19), (20), and (2) sampled. (d) Control laws (14), (19), (20), and (2) implemented with the exponential holder. (e) Control laws as in (d), but with $\delta=460 \mu \mathrm{s}$
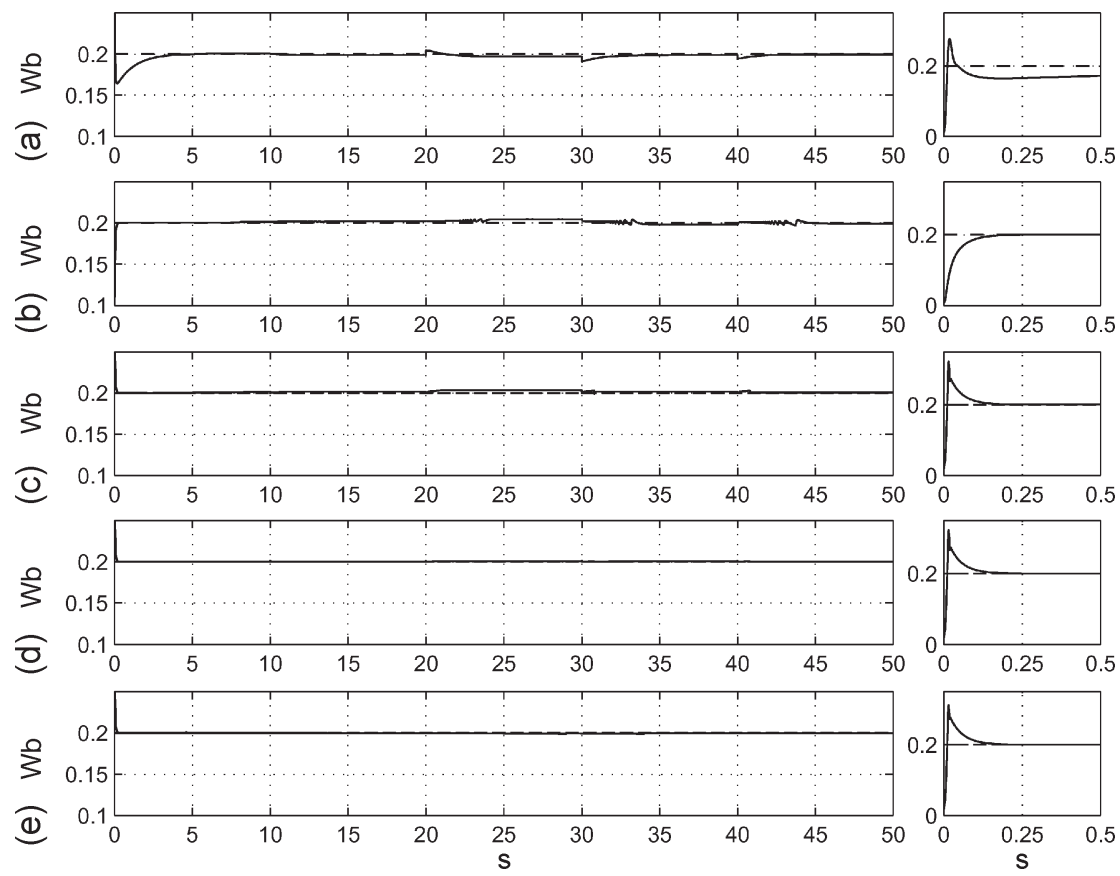

Fig. 2. (Solid) Estimated square flux modulus $\left\|\hat{\Phi}_{k}\right\|^{2}$ and (dashed) reference $\Phi_{r, k}$. (a) FOC with observer. (b) Digital control and observer (Euler approximation). (c) Control laws (14), (19), (20), and (2) sampled. (d) Control laws (14), (19), (20), and (2) implemented with the exponential holder. (e) Control laws as in (d), but with $\delta=460 \mu \mathrm{s}$. (Right) Zoom from 0 to $0.5 \mathrm{~s}$ of the same graphics on the left.

d) control law given by (14), (19), (20), and (2) implemented as explained in Section IV-B;

e) control law as in d), but with a doubled sampling period $\delta=460 \mu \mathrm{s}$.

In Figs. 1 and 2, the simulation results for these five schemes are compared. The rotor angular velocity reference increases from 0 to $100 \mathrm{rad} / \mathrm{s}$ in the first $20 \mathrm{~s}$ and, then, is piecewise constant and equal to $70 \mathrm{rad} / \mathrm{s}$ for $t \in[30,40)$ and to $40 \mathrm{rad} / \mathrm{s}$ for $t \in[40,50]$. The flux modulus reference has a constant value of $0.2 \mathrm{~Wb}^{2}$. The other variables have not been reported due to space limitations. Note that the behaviors of the controllers c) and d) are practically coincident, at least for the chosen sampling rate. This means that the simple sampling of (2) is an effective solution, which avoids the use of analog devices, at least for sufficiently small sampling periods $\delta$. In fact, it is obvious that, as $\delta \rightarrow 0$, one recovers the continuous behavior. 

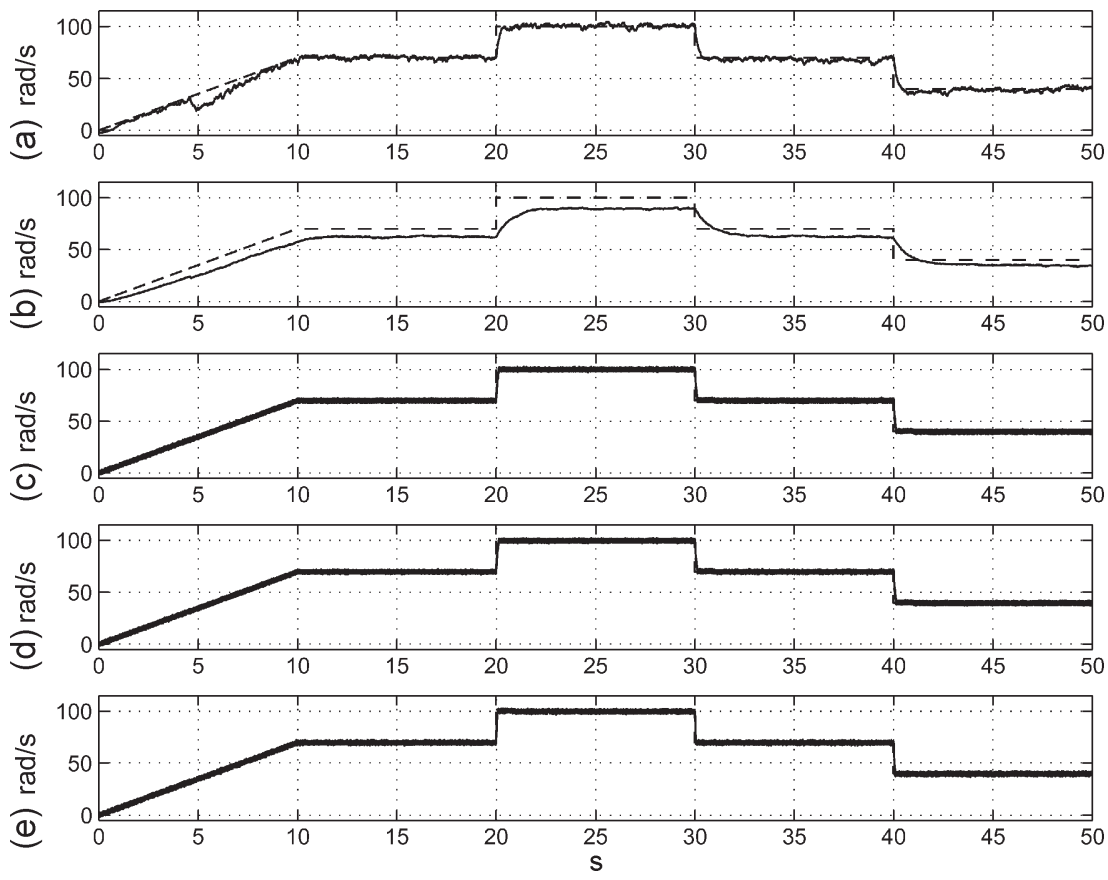

Fig. 3. (Solid) Rotor angular velocity $\omega_{k}$ and (dashed) reference $\omega_{r, k}$ in the case of measurement noise. (a) FOC with observer. (b) Digital control and observer (Euler approximation). (c) Control laws (14), (19), (20), and (2) sampled. (d) Control laws (14), (19), (20), and (2) implemented with the exponential holder. (e) Control laws as in (d), but with $\delta=460 \mu \mathrm{s}$.
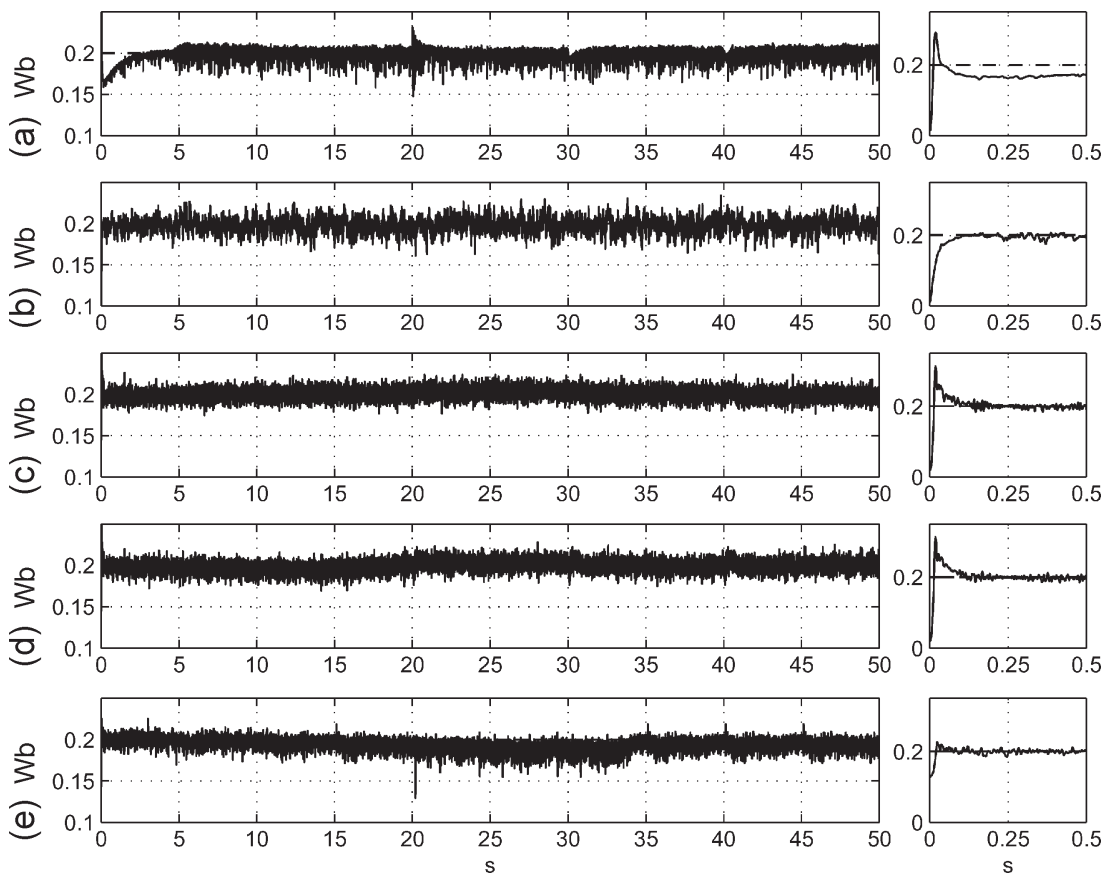

Fig. 4. (Solid) Estimated square flux modulus $\left\|\hat{\Phi}_{k}\right\|^{2}$ and (dashed) reference $\Phi_{r, k}$ in the case of measurement noise. (a) FOC with observer. (b) Digital control and observer (Euler approximation). (c) Control laws (14), (19), (20), and (2) sampled. (d) Control laws (14), (19), (20), and (2) implemented with the exponential holder. (e) Control laws as in (d), but with $\delta=460 \mu \mathrm{s}$. (Right) Zoom from 0 to $0.5 \mathrm{~s}$ of the same graphics on the left.

Anyway, on the basis of the comments already done, there is no theoretical assurance in case c) that the digital representation is still accurate as $\delta$ increases. It is also worth noting that the controller d) performs better with respect to the sampled FOC or a digital controller determined on the basis of simpler discrete-time representations, such as the Euler approximation. The controller e) shows a behavior similar to that in d), because the exact continuous reproduction is ensured at the sampling time by the sampled closed model, in accordance with the theory. Note that nothing can be ensured for the intersamplings.

As final consideration, because the implementation of these control laws requires the knowledge of the angular velocity, whose measurement is usually quite poor in practice, a degeneration of the controller performance is expected due to measurement noise. In order to evaluate this important aspect, in Figs. 3 and 4, we have considered the previous controllers 


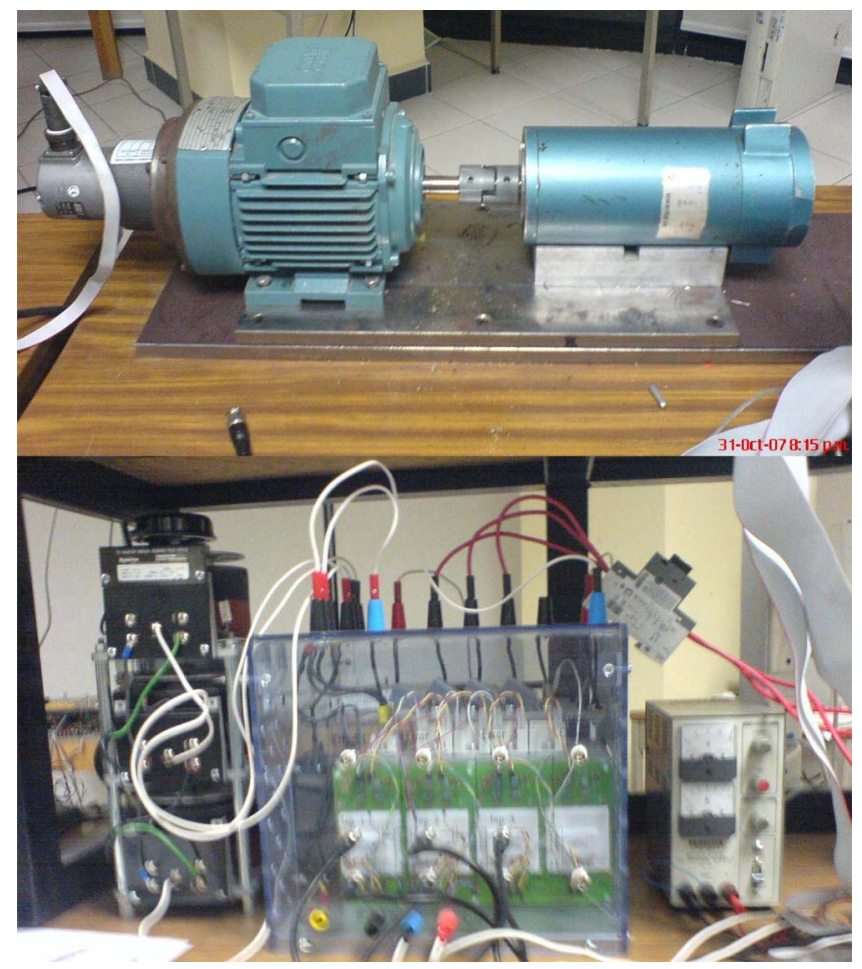

Fig. 5. Experimental setup.

with measurement noise. In this case, the performance of the controllers c) and d) are practically the same. However, the degradation in performance of controller b) and the sensitivity of controller a) with respect to load torque variations suggest the superiority of the proposed controller d).

\section{B. Experimental Results}

The experimental setup, shown in Fig. 5, consists of an IM with the same characteristics given in the Section V-A. The power module is a three-phase inverter Semikron IGBT Power Electronics Teaching System, which incorporates a three-phase rectifier fed by a three-phase ac variator. The control and PWM generation are implemented with a DSP board (Dspace 1104). The signals from the phase currents are acquired by analog-todigital converters included in the DSP board.

The motor is loaded by a dc generator connected to a variable-power resistor. In the tests, we reproduced a load torque $T$ of $0.2 \mathrm{Nm}$, with a variation to $1 \mathrm{Nm}$ at $t=4.6 \mathrm{~s}$, as in the simulations.

The motor position and speed are measured by an optical encoder with $1024 \mathrm{ppr}$ (pulses per revolution) of resolution and filtered by a first-order Butterworth low-pass filter having an $8 \mathrm{rad} / \mathrm{s}$ edge frequency, to attenuate the measurement noise. The stator phase currents are measured by Hall-type sensors.

On the base of the time constraints of the implementation, the sampling period has been fixed equal to $\delta=230 \mu \mathrm{s}$, which is a typical value in applications, as already observed. However, we recall that an advantage of obtaining an exact discrete-time model is that the sampling period to be used in the implementation of the control law can be high, at least theoretically. Other aspects, such as actuators, data acquisition, etc., limit in practice its value.
Fig. 6 shows the output tracking results. The references have been chosen as in Section V-A. The results are similar to those in Section V-A, showing slight deviations corresponding to step variations. These differences can be attributed to the highfrequency effects of the unmodeled dynamics of the actuator, not considered in the mathematical model. Finally, Fig. 7 shows the experimental behaviors of the components $i_{s, a, k}$ and $i_{s, b, k}$ of the stator current vector $I_{s, k}$ and those of the input voltages $u_{a, k}$ and $u_{b, k}$.

\section{CONCLUSION}

In this paper, an exact sampled data representation of the IM has been obtained. This sampled data model has been determined by means of a particular controller which induces a sampled closed representation for the original continuous time model. On the basis of the exact sampled data model, an observer-based controller, which guarantees asymptotic reference tracking for the velocity and flux in the presence of an unknown load torque, has been designed. Some interesting issues, such as the robustness of the controller with respect to parameter variations, determination of the sampling period, and modeling of the actuator's dynamics, are currently under study.

\section{APPENDIX I \\ SAMPLED DYNAMICS OF SySTEM (8)}

Integrating (5) on the time interval $[k \delta, t), t<(k+1) \delta$, one obtains

$$
\begin{aligned}
\left(\begin{array}{c}
\Phi(t) \\
I(t)
\end{array}\right)= & \left(\begin{array}{ll}
\varphi_{1}(t-k \delta) \mathbb{I}_{2 \times 2} & \varphi_{2}(t-k \delta) \mathbb{I}_{2 \times 2} \\
\varphi_{3}(t-k \delta) \mathbb{I}_{2 \times 2} & \varphi_{4}(t-k \delta) \mathbb{I}_{2 \times 2}
\end{array}\right)\left(\begin{array}{c}
\Phi_{k} \\
I_{k}
\end{array}\right) \\
& +\left(\begin{array}{c}
\zeta_{1}(t-k \delta) \mathbb{I}_{2 \times 2} \\
\zeta_{2}(t-k \delta) \mathbb{I}_{2 \times 2}
\end{array}\right) v_{k} \\
\omega(t)= & \omega_{k}+\mu \int_{k \delta}^{t} I^{T} \Im \Phi d \tau-\frac{C_{k}}{J}(t-k \delta) \\
\theta(t)= & \theta_{k}+\int_{k \delta}^{t} \omega(\tau) d \tau
\end{aligned}
$$

where, denoting "sinh" and "cosh" by "sinh" and "cosh," respectively

$$
\begin{aligned}
& \varphi_{1}(t)=e^{-\rho t}\left[\frac{\gamma-\alpha}{2 \omega_{0}} \sinh \omega_{0} t+\cosh \omega_{0} t\right] \\
& \varphi_{2}(t)=\frac{\alpha L_{m}}{\omega_{0}} e^{-\rho t} \sinh \omega_{0} t \\
& \varphi_{3}(t)=\frac{\alpha \beta}{\omega_{0}} e^{-\rho t} \sinh \omega_{0} t \\
& \varphi_{4}(t)=e^{-\rho t}\left[-\frac{\gamma-\alpha}{2 \omega_{0}} \sinh \omega_{0} t+\cosh \omega_{0} t\right] \\
& \zeta_{1}(t)=\frac{L_{m}}{R_{s}}\left[1-e^{-\rho t}\left[\frac{\rho}{\omega_{0}} \sinh \omega_{0} t+\cosh \omega_{0} t\right]\right] \\
& \zeta_{2}(t)=\frac{1}{R_{s}}\left[1-e^{-\rho t}\left[\frac{1}{\omega_{0}}\left(\rho-\frac{R_{s}}{L_{\sigma}}\right) \sinh \omega_{0} t+\cosh \omega_{0} t\right]\right]
\end{aligned}
$$



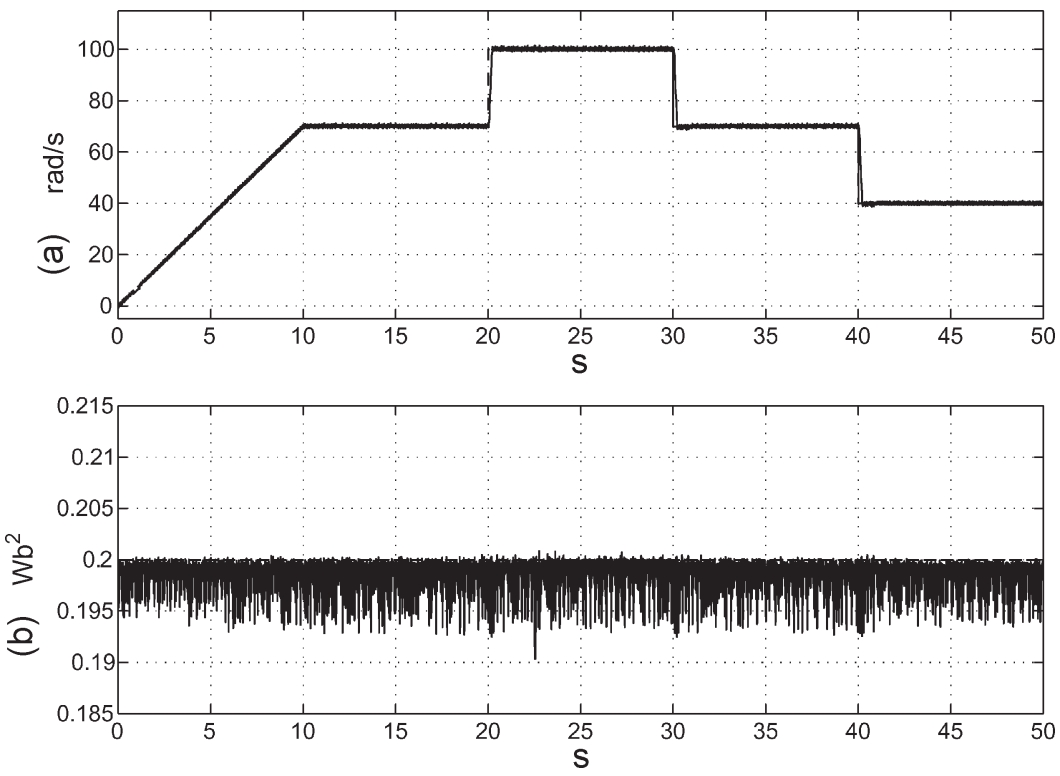

Fig. 6. (a) (Solid) Rotor angular velocity $\omega_{k}$ and (dashed) reference $\omega_{r, k}$. (b) (Solid) Estimated square flux modulus $\left\|\hat{\Phi}_{k}\right\|^{2}$ and (dashed) reference $\Phi_{r, k}$.
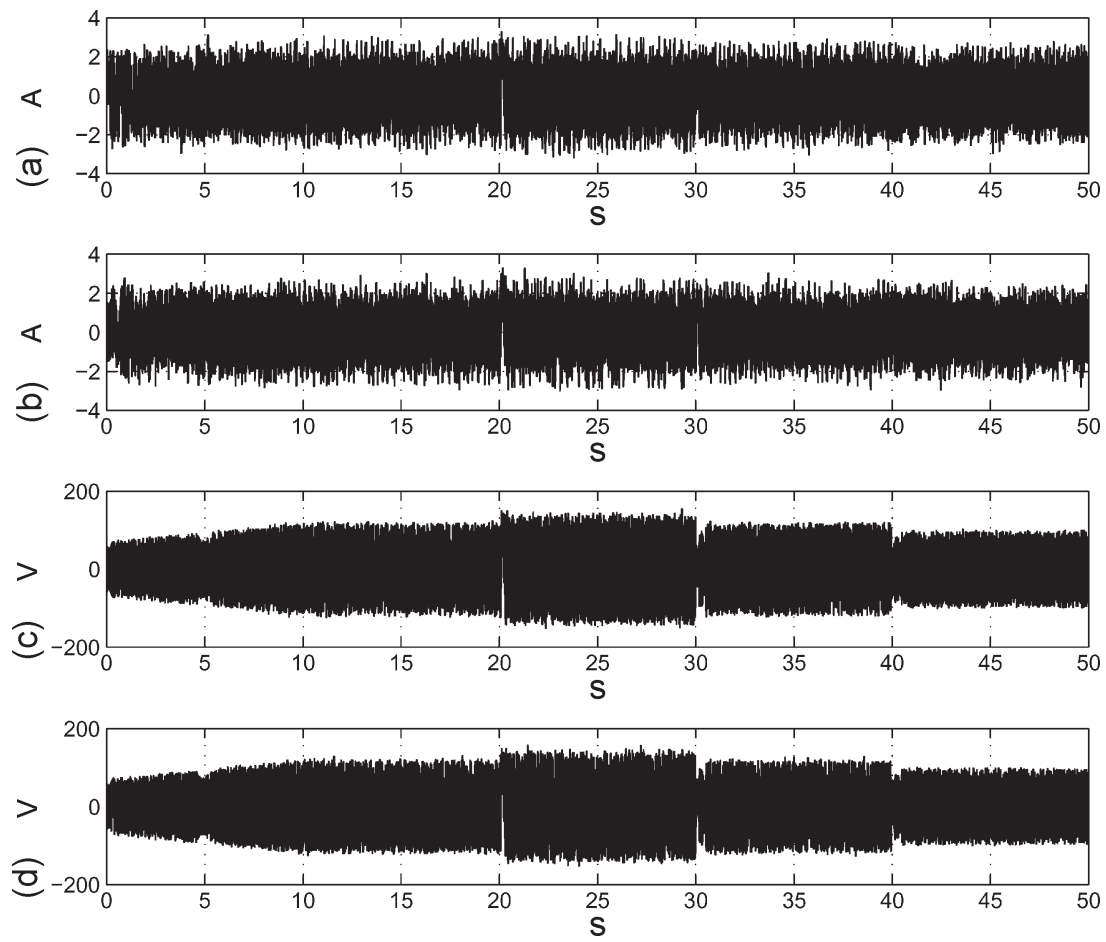

Fig. 7. Stator current components: (a) $i_{s, a, k}$ and (b) $i_{s, b, k}$. Control input voltages: (c) $u_{a, k}$ and (d) $u_{b, k}$.

with $\rho=(\alpha+\gamma) / 2=\left(L_{s} R_{r} / L_{r}+R_{s}\right) /\left(2 L_{\sigma}\right)$ and

$$
\begin{aligned}
\omega_{0} & =\sqrt{(\gamma-\alpha)^{2}+4 \alpha^{2} \beta L_{m}} / 2=\sqrt{\rho^{2}-\alpha R_{s} / L_{\sigma}} \\
\gamma-\alpha & =\left(R_{s}+\left(2 L_{m}^{2}-L_{s} L_{r}\right) R_{r} / L_{r}^{2}\right) / L_{\sigma} .
\end{aligned}
$$

Note that, for our case, $\gamma-\alpha>0$.
For the expressions of $\omega(t)$ and $\theta(t)$, one works out

$$
\begin{aligned}
\omega(t)= & \omega_{k}+\nu_{1}(t-k \delta) I_{k}^{T} \Im \Phi_{k}+\nu_{2}(t-k \delta) \Phi_{k}^{T} \Im v_{k} \\
& +\nu_{3}(t-k \delta) I_{k}^{T} \Im v_{k}-\frac{C_{k}}{J}(t-k \delta) \\
\theta(t)= & \theta_{k}+(t-k \delta) \omega_{k}+\nu_{4}(t-k \delta) I_{k}^{T} \Im \Phi_{k} \\
& +\nu_{5}(t-k \delta) \Phi_{k}^{T} \Im v_{k}+\nu_{6}(t-k \delta) I_{k}^{T} \Im v_{k} \\
& -\frac{1}{2}(t-k \delta)^{2} \frac{C_{k}}{J}
\end{aligned}
$$


where

$$
\begin{aligned}
\nu_{1}(t)= & \frac{\mu}{\rho} e^{-\rho t} \sinh \rho t \\
\nu_{2}(t)= & \frac{\mu}{\alpha R_{s}} e^{-\rho t}\left[\frac{\gamma-\alpha}{2}\left(\frac{1}{\omega_{0}} \sinh \omega_{0} t-\frac{1}{\rho} \sinh \rho t\right)\right. \\
& \left.\quad+\cosh \omega_{0} t-\cosh \rho t\right] \\
\nu_{3}(t)= & \frac{\mu L_{m}}{R_{s}} e^{-\rho t}\left[\frac{1}{\omega_{0}} \sinh \omega_{0} t-\frac{1}{\rho} \sinh \rho t\right] \\
\nu_{4}(t)= & \frac{\mu^{2}}{\alpha+\gamma}\left[t-\frac{e^{-\rho t}}{\rho} \sinh \rho t\right] \\
\nu_{5}(t)= & \frac{\mu^{2} L_{\sigma}}{\alpha^{2} R_{s}^{2}}\left[\gamma-e^{-\rho t}\left[\frac{(\gamma-\alpha) \gamma+2 \alpha^{2} \beta L_{m}}{2 \omega_{0}} \sinh \omega_{0} t\right.\right. \\
& -\frac{\mu^{2} L_{m}}{R_{s}} \frac{e^{-\rho t}}{\rho^{2}} \sinh \rho t . \\
& \frac{\mu^{2}}{(\alpha+\gamma) R_{s}}\left[\frac{e^{-\rho t}}{\rho} \sinh \rho t+\frac{\gamma}{\alpha} t\right] \\
\nu_{6}(t)= & \frac{\mu^{2} L_{m} L_{\sigma}}{\alpha R_{s}^{2}}\left[1-e^{-\rho t}\left[\frac{\alpha+\gamma}{\omega_{0}} \sinh \omega_{0} t+\cosh \omega_{0} t\right]\right]
\end{aligned}
$$

For $t=(k+1) \delta$, we finally obtain the expressions in (6) with

$$
\begin{array}{rlll}
a_{11}=\varphi_{1}(\delta) & a_{12}=\varphi_{2}(\delta) & a_{21}=\varphi_{3}(\delta) & a_{22}=\varphi_{4}(\delta) \\
b_{1}=\zeta_{1}(\delta) & b_{2}=\zeta_{2}(\delta) & \eta_{1}=\nu_{1}(\delta) & \eta_{2}=\nu_{2}(\delta) \\
\eta_{3}=\nu_{3}(\delta) & \kappa_{1}=\nu_{4}(\delta) & \kappa_{2}=\nu_{5}(\delta) & \kappa_{3}=\nu_{6}(\delta)
\end{array}
$$

Note that the coefficient $b_{1}$ is always positive for all $\delta>0$, as can be verified by plotting $b_{1}$ as a function of $\delta$. Note also that the matrix $A_{d}$ in (7) can be easily transformed into the matrix $\operatorname{diag}\{A, A\}$, where

$$
A=\left(\begin{array}{ll}
a_{11} & a_{12} \\
a_{21} & a_{22}
\end{array}\right) .
$$

$A$ is a nonsingular matrix with determinant $|A|=e^{-\rho \delta}$ and with characteristic polynomial $p(\lambda)=\lambda^{2}+a_{1} \lambda+a_{0}, a_{1}=$ $-2 e^{-\rho \delta} \cosh \omega_{0} \delta$, and $a_{0}=|A|=e^{-\rho \delta}$. Applying Jury's criterion [4], one gets that all its roots are inside the unit circle if and only if $a_{0}=e^{-\rho \delta}<1$ and $a_{0} \pm a_{1}+1=$ $e^{-\rho \delta} \pm 2 e^{-\rho \delta} \cosh \omega_{0} \delta+1>0$. Because $\rho>0$ and $e^{-\rho \delta} \pm$ $2 e^{-\rho \delta} \cosh \omega_{0} \delta+1$ is always positive, one easily concludes that $A_{d}$ is Schur for every choice of the sampling period $\delta>0$.

Finally, considering the matrix $\mathcal{R}$ given by (15), one works out that the determinant is

$$
\operatorname{det} \mathcal{R}=\left(a_{11} \eta_{3}-a_{12} \eta_{2}\right)^{4} / 16
$$

where

$$
\begin{array}{r}
a_{11} \eta_{3}-a_{12} \eta_{2}=\frac{\mu L_{m}}{\omega_{0} R_{s}} e^{-2 \rho \delta}\left(\sinh \omega_{0} \delta \cosh \rho \delta\right. \\
\left.-\frac{\omega_{0}}{\rho} \cosh \omega_{0} \delta \sinh \rho \delta\right) \\
=\frac{\mu L_{m}}{\omega_{0} R_{s}} d_{R, \delta}\left(\tanh \omega_{0} \delta-\frac{\omega_{0}}{\rho} \tanh \rho \delta\right)
\end{array}
$$

with

$$
d_{\mathcal{R}, \delta}=e^{-2 \rho \delta} \cosh \omega_{0} \delta \cosh \rho \delta .
$$

Here, $\tanh \omega_{0} \delta / \tanh \rho \delta-\omega_{0} / \rho$ is a positive increasing function of $\delta$, and $d_{\mathcal{R}, \delta}$ is different from zero for $\delta \neq 0$.

\section{APPENDIX II}

\section{SAMPLING OF $(a, b)$ ROTOR FLUX VECTOR ANGULAR POSITION}

In the stator reference frame $(a, b)$, the rotor flux at time $k \delta$ can be obtained from (4)

$$
\begin{aligned}
\phi_{r, k} & =\left(\begin{array}{c}
\phi_{r, a, k} \\
\phi_{r, b, k}
\end{array}\right)=e^{p \theta_{k} \Im} \Phi_{k}, \quad \Phi_{k}=\left(\begin{array}{c}
\phi_{a, k} \\
\phi_{b, k}
\end{array}\right) \\
& =\left(\begin{array}{cc}
\cos p \theta_{k} & -\sin p \theta_{k} \\
\sin p \theta_{k} & \cos p \theta_{k}
\end{array}\right)\left(\begin{array}{c}
\phi_{a, k} \\
\phi_{b, k}
\end{array}\right) \\
& =\left(\begin{array}{c}
\phi_{a, k} \cos p \theta_{k}-\phi_{b, k} \sin p \theta_{k} \\
\phi_{a, k} \sin p \theta_{k}+\phi_{b, k} \cos p \theta_{k}
\end{array}\right) .
\end{aligned}
$$

Hence, the angular position of $\phi_{r, k}$ is given by

$$
\begin{aligned}
\theta_{\Phi, k} & =\arctan \frac{\phi_{r, b, k}}{\phi_{r, a, k}}=\frac{\phi_{a, k} \sin p \theta_{k}+\phi_{b, k} \cos p \theta_{k}}{\phi_{a, k} \cos p \theta_{k}-\phi_{b, k} \sin p \theta_{k}} \\
& =p \theta_{k}+\arctan \frac{\phi_{b, k}}{\phi_{a, k}}
\end{aligned}
$$

where some trigonometric manipulations have been performed.

Moreover, considering that

$$
\phi_{r, k}=\sqrt{\Phi_{k}^{T} \Phi_{k}}\left(\begin{array}{c}
\cos \theta_{\Phi, k} \\
\sin \theta_{\Phi, k}
\end{array}\right)
$$

one gets

$$
\begin{aligned}
\Phi_{k} & =e^{-p \theta_{k} \Im} \phi_{r, k} \\
& =\left(\begin{array}{cc}
\cos p \theta_{k} & \sin p \theta_{k} \\
-\sin p \theta_{k} & \cos p \theta_{k}
\end{array}\right) \sqrt{\Phi_{k}^{T} \Phi_{k}}\left(\begin{array}{c}
\cos \theta_{\Phi, k} \\
\sin \theta_{\Phi, k}
\end{array}\right) \\
& =\sqrt{e_{2, k}+\Phi_{r, k}} \vartheta_{k}
\end{aligned}
$$

with $\Phi_{k}^{T} \Phi_{k}=e_{2, k}+\Phi_{r, k}$ and where we have defined

$$
\vartheta_{k}=\left(\begin{array}{c}
\cos \left(\theta_{\Phi, k}-p \theta_{k}\right) \\
\sin \left(\theta_{\Phi, k}-p \theta_{k}\right)
\end{array}\right) .
$$




\section{APPENDIX III \\ ZERO DYNAMICS}

The difficulties in studying the stability of the whole controlled system are due to the fact that the strict feedback form [20], as that of the IM dynamics, is lost under sampling. To the best of the authors' knowledge, there are not general results on the stability of such sampled systems. In the following, we analyze the so-called zero dynamics and we give some results. The system dynamics can be rewritten using the new state variables $\left(\begin{array}{lllll}e_{1, k} & e_{2, k} & I_{k}^{T} & \theta_{\Phi, k} & \theta_{k}\end{array}\right)^{T}$, where the first two variables are exactly the outputs of the system and where the "complement" of this state transformation is given by the current $I_{k}$, the rotor flux angular position $\theta_{\Phi, k}$ [see (26)], and the rotor angular position $\theta_{k}$. The zero dynamics are the dynamics of the complement variables when the outputs are zero.

The current dynamics [the second of (6)]

$$
\begin{aligned}
I_{k+1} & =a_{22} I_{k}+\mathcal{W}_{k} \\
\mathcal{W}_{k} & =a_{21} \sqrt{e_{2, k}+\Phi_{r, k}} \vartheta_{k}+b_{2} \mathcal{T}_{k}^{-1} \mathcal{V}_{k}
\end{aligned}
$$

have eigenvalues inside the unit circle $\left(a_{22} \in(0,1)\right.$ for all $\delta>0$ - see Appendix I), so that these dynamics are bounded input-bounded output. Unfortunately, it is not evident how to show that the "input" $\mathcal{W}_{k}$ is bounded. In fact, while $\vartheta_{k}$ is given by bounded (trigonometric) functions, $\Phi_{r, k}$ is bounded, and $e_{2, k}$ tends to zero, it is easy to check that $v_{k}=\mathcal{T}_{k}^{-1} \mathcal{V}_{k}$ depends explicitly on $I_{k}$. A possible solution to circumvent this difficulty is to show the existence of a steady-state function $I_{r, k}$ for $I_{k}$. On this ground, we first get rid of $u_{k}$ between the flux and current equations [first two equations of (6)], considering the variable $\bar{I}_{k}=b_{2} \Phi_{k}-b_{1} I_{k}$. One easily obtains

$$
\bar{I}_{k+1}=\bar{\lambda} \bar{I}_{k}+\bar{b} \Phi_{k}, \quad \Phi_{k}=\sqrt{e_{2, k}+\Phi_{r, k}} \vartheta_{k}
$$

where

$$
\begin{aligned}
& \bar{\lambda}=a_{22}-a_{12} b_{2} / b_{1} \\
& \bar{b}=\left(a_{11}-a_{22}\right) b_{2}-a_{21} b_{1}+a_{12} b_{2}^{2} / b_{1} .
\end{aligned}
$$

It is possible to check that $|\bar{\lambda}|<1$ (see Appendix I). These linear dynamics are bounded input-bounded output, forced by a bounded input $\Phi_{k}$ ( $e_{2, k}$ goes to zero, $\Phi_{r, k}$ is bounded by assumption, and $\vartheta_{k}$ has trigonometric - sin and cosine-components). Hence, $\bar{I}_{k}$ is bounded and therefore also

$$
I_{k}=\frac{b_{2}}{b_{1}} \Phi_{k}-\frac{1}{b_{1}} \bar{I}_{k}
$$

is bounded. Moreover, there exists a steady state

$$
\bar{I}_{r, k}=\sum_{j=0}^{k-1} \bar{\lambda}^{k-j-1} \bar{b} \sqrt{\Phi_{r, j}} \vartheta_{j}
$$

solution of the linear equation

$$
\bar{I}_{r, k+1}=\bar{\lambda} \bar{I}_{r, k}+\bar{b} \sqrt{\Phi_{r, k}} \vartheta_{k} .
$$

It is easy to see that $\bar{I}_{k}$ converges to $\bar{I}_{r, k}$. In fact

$$
\bar{I}_{k+1}-\bar{I}_{r, k+1}=\bar{\lambda}\left(\bar{I}_{k}-\bar{I}_{r, k}\right)+\bar{b}\left(\sqrt{e_{2, k}+\Phi_{r, k}}-\sqrt{\Phi_{r, k}}\right) \vartheta_{k}
$$

$|\bar{\lambda}|<1$, and $\Phi_{k}$ converges to $\sqrt{\Phi_{r, k}} \vartheta_{k}$ (because $e_{2, k}$ tends to zero). Therefore, $I_{k}$ converges asymptotically to

$$
\begin{aligned}
I_{r, k} & =\frac{b_{2}}{b_{1}} \sqrt{\Phi_{r, k}} \vartheta_{k}-\frac{1}{b_{1}} \bar{I}_{r, k} \\
& =\frac{b_{2}}{b_{1}} \sqrt{\Phi_{r, k}} \vartheta_{k}-\frac{\bar{b}}{b_{1}} \sum_{j=0}^{k-1} \bar{\lambda}^{k-j-1} \sqrt{\Phi_{r, j}} \vartheta_{j} .
\end{aligned}
$$

Finally, the dynamics of $\theta_{\Phi, k+1}$ and $\theta_{k}$ may be unstable, namely, $\theta_{\Phi, k+1}$ and $\theta_{k}$ may go to infinity. This is physically admissible because they refer to the flux and rotor angular positions [in practice, they take values in $[-\pi, \pi)]$, and is mathematically acceptable because $\theta_{\Phi, k}$ and $\theta_{k}$ enter in the other equations (those of $I_{k}$ ) through $\vartheta_{k}$, which is bounded.

\section{ACKNOWLEDGMENT}

The authors would like to thank the anonymous referees for their valuable comments which improved this paper.

\section{REFERENCES}

[1] B. Akin, U. Orguner, A. Ersak, and M. Ehsani, "Simple derivative-free nonlinear state observer for sensorless AC drives," IEEE/ASME Trans. Mechatronics, vol. 11, no. 5, pp. 634-643, Oct. 2006.

[2] A. Y. Alanis, E. N. Sanchez, and A. G. Loukianov, "Discrete-time adaptive backstepping nonlinear control via high-order neural networks," IEEE Trans. Neural Netw., vol. 18, no. 4, pp. 1185-1195, Jul. 2007.

[3] F. Alonge, F. D'Ippolito, G. Giardina, and T. Scaffidi, "Design and low-cost implementation of an optimally robust reduced-order rotor flux observer for induction motor control," IEEE Trans. Ind. Electron., vol. 54, no. 6, pp. 3205-3216, Dec. 2007.

[4] K. J. Åström and B. Wittenmark, Computer-Controlled Systems. Englewood Cliffs, NJ: Prentice-Hall, 1990.

[5] A. Benchaib, A. Rachid, E. Audrezet, and M. Tadjine, "Real-time sliding-mode observer and control of an induction motor," IEEE Trans. Ind. Electron., vol. 46, no. 1, pp. 128-138, Feb. 1999.

[6] F. Blaschke, "The principle of field orientation as applied to the new transvector closed-loop control system for rotating field machines," Siemens Rev., vol. 39, no. 5, pp. 217-220, 1972.

[7] W. M. Caminhas, R. H. C. Takahashi, P. L. D. Peres, and H. M. F. Tavares, "Discrete time sliding modes observers for induction motors," in Proc. IEEE Int. Conf. Control Appl., Dearborn, MI, Sep. 15-18, 1996, pp. 314-319.

[8] B. Castillo-Toledo and S. Di Gennaro, "On the nonlinear ripple free sampled-data robust regulator," Eur. J. Control, vol. 8, no. 1, pp. 44-55, 2002.

[9] B. Castillo-Toledo and G. Obregón-Pulido, "Guaranteeing asymptotic zero intersampling tracking error via discretized regulator and exponential holder for nonlinear systems," J. Appl. Res. Technol., vol. 1, no. 3, pp. 203-214, 2003.

[10] B. Castillo-Toledo, S. Di Gennaro, A. G. Loukianov, and J. Rivera, "On the discrete-time modeling and control of induction motors with sliding modes," in Proc. ACC, Boston, MA, 2004, pp. 2598-2602.

[11] B. Castillo-Toledo, S. Di Gennaro, A. G. Loukianov, and J. Rivera, "Discrete time sliding mode control with application to induction motors," Automatica. to be published.

[12] G.-W. Chang, G. Espinosa-Perez, E. Mendes, and R. Ortega“"Tuning rules for the PI gains of field-oriented controllers of induction motors," IEEE Trans. Ind. Electron., vol. 47, no. 3, pp. 592-602, Jun. 2000.

[13] M. N. Cirstea and A. Dinu, "A VHDL holistic modeling approach an FPGA implementation of a digital sensorless induction motor control scheme," IEEE Trans. Ind. Electron., vol. 54, no. 4, pp. 1853-1864, Aug. 2007.

[14] M. Comanescu and L. Xu, "An improved flux observer based on PLL frequency estimator for sensorless vector control of induction motors," IEEE Trans. Ind. Electron., vol. 53, no. 1, pp. 50-56, Feb. 2006. 
[15] S. Di Gennaro, S. Monaco, and D. Normand-Cyrot, "Nonlinear digital scheme for attitude tracking," AIAA J. Guid. Control Dyn., vol. 22, no. 3, pp. 467-477, 1999.

[16] P. Di Giamberardino, S. Monaco, and D. Normand-Cyrot, "On equivalence and feedback equivalence to finitely computable sampled models," in Proc. 45th Conf. Decision Control, San Diego, CA, 2006, pp. 5869-5874.

[17] F. G. Garashchenko and I. A. Kutsenko, "Problems of practical stability and stabilization of motion of discrete-time systems," J. Math. Sci., vol. 66, no. 5, pp. 2538-2543, 1993.

[18] N. S. Gehlot and P. J. Alsina, "A discrete model of induction motors for real-time control applications," IEEE Trans. Ind. Electron., vol. 40, no. 3, pp. 317-325, Jun. 1993.

[19] L. U. Gokdere, M. A. Simaan, and C. W. Brice, "Passivity-based control of saturated induction motors," IEEE Trans. Ind. Electron., vol. 48, no. 4, pp. 870-872, Aug. 2001.

[20] H. F. Ho, Y. K. Wong, and A. B. Rad, "Adaptive fuzzy approach for a class of uncertain nonlinear systems in strict-feedback form," ISA Trans., vol. 47, no. 3, pp. 286-299, Jul. 2008.

[21] S. H. Jeon, K. K. Oh, and J. Y. Choi, "Flux observer with online tuning of stator and rotor resistances for induction motors," IEEE Trans. Ind. Electron., vol. 49, no. 3, pp. 653-664, Jun. 2002.

[22] A. Isidori, Nonlinear Control Systems, 3rd ed. London, U.K.: SpringerVerlag, 1995.

[23] P. C. Krause, O. Wasynczuk, and S. D. Sudhoff, Analysis of Electric Machinery. New York: IEEE Press, 1995.

[24] C. Lascu and G.-D. Andreescu, "Sliding-mode observer and improved integrator with DC-offset compensation for flux estimation in sensorlesscontrolled induction motors," IEEE Trans. Ind. Electron., vol. 53, no. 3, pp. 785-794, Jun. 2006.

[25] C.-M. Lin and C.-F. Hsu, "Recurrent-neural-network-based adaptivebackstepping control for induction servomotors," IEEE Trans. Ind. Electron., vol. 52, no. 6, pp. 1677-1684, Dec. 2005.

[26] A. G. Loukianov, J. Rivera, and J. M. Cañedo, "Discrete-time sliding mode control of an induction motor," in Proc. 15th IFAC World Congr., Barcelona, Spain, 2002, pp. 2693-2698.

[27] R. Marino, S. Peresada, and P. Valigi, "Adaptive input-output linearizing control of induction motors," IEEE Trans. Autom. Control, vol. 38, no. 2, pp. 208-221, Feb. 1993.

[28] S. Monaco and D. Normand-Cyrot, "On the sampling of linear analytic control systems," in Proc. 24th Conf. Decision Control, 1985, pp. 1467-1472.

[29] S. Monaco and D. Normand-Cyrot, "On the linearizing feedback in nonlinear sampled data control schemes," in Proc. 25th Conf. Decision Control, 1986, pp. 2056-2060.

[30] S. Monaco and D. Normand-Cyrot, "Zero dynamics of sampled nonlinear systems," Syst. Control Lett., vol. 11, no. 3, pp. 229-234, 1988.

[31] S. Monaco and D. Normand-Cyrot, Multirate Sampling and Zero Dynamics: From Linear to Nonlinear, C. Byrnes and A. Kurzhansky, Eds. Boston, MA: Birkaüser, 1991, pp. 200-213.

[32] S. Monaco, P. Di Giamberardino, and D. Normand-Cyrot, "Digital control through finite feedback discretizability," in Proc. IEEE Int. Conf. Robot. Autom., Minneapolis, MN, 1996, pp. 3141-3146.

[33] S. Monaco and D. Normand-Cyrot, "Minimum phase nonlinear discretetime systems and feedback stabilization," in Proc. 26th Conf. Decision Control, 1987, pp. 979-986.

[34] S. Monaco and D. Normand-Cyrot, "A unified representation for nonlinear discrete-time and sampled dynamics," J. Math. Syst., Estimation, Control, vol. 7, no. 4, pp. 477-503, 1997.

[35] S. Monaco, D. Normand-Cyrot, and A. Chelouah, "Digital nonlinear speed regulation of a synchronous motor," Avtomatika i Telemekhanika, vol. 58, no. 6, pp. 143-157, 1997.

[36] S. Monaco, D. Normand-Cyrot, and A. Chelouah, "Digital nonlinear speed regulation of a synchronous motor," Autom. Remote Control, vol. 58, no. 6, pp. 1003-1016, 1997.

[37] S. Monaco and D. Normand-Cyrot, "Issues on nonlinear digital systems," Eur. J. Control, vol. 7, pp. 160-178, 2001.

[38] S. Monaco and D. Normand-Cyrot, "Advanced tools for nonlinear sampled-data systems' analysis and control," Eur. J. Control, vol. 13, no. 2/3, pp. 221-241, 2007.

[39] A. B. Proca and A. Keyhani, "Sliding-mode flux observer with online rotor parameter estimation for induction motors," IEEE Trans. Ind. Electron., vol. 54, no. 2, pp. 716-723, Apr. 2007.

[40] R. Shahnazi, H. M. Shanechi, and N. Pariz, "Position control of induction and DC servomotors: A novel adaptive fuzzy PI sliding mode control," IEEE Trans. Energy Convers., vol. 23, no. 1, pp. 138-147, Mar. 2008.
[41] S. Suwankawin and S. Sangwongwanich, "Design strategy of an adaptive full-order observer for speed-sensorless induction-motor drives-tracking performance and stabilization," IEEE Trans. Ind. Electron., vol. 53, no. 1, pp. 96-119, Feb. 2006.

[42] Y. Tan, J. Chang, and H. Tan, "Adaptive backstepping control and friction compensation for AC servo with inertia and load uncertainties," IEEE Trans. Ind. Electron., vol. 50, no. 5, pp. 944-952, Oct. 2003.

[43] A. V. Topalov, G. L. Cascella, V. Giordano, F. Cupertino, and O. Kaynak, "Sliding mode neuro-adaptive control of electric drives," IEEE Trans. Ind. Electron., vol. 54, no. 1, pp. 671-679, Feb. 2007.

[44] J. C. Travieso-Torres and M. A. Duarte-Mermoud, "Two simple and novel SISO controllers for induction motors based on adaptive passivity," ISA Trans., vol. 47, no. 1, pp. 60-79, Jan. 2008.

[45] V. I. Utkin, "Sliding mode control design principles and applications to electric drives," IEEE Trans. Ind. Electron., vol. 40, no. 1, pp. 23-36, Feb. 1993.

[46] P. Vaclavek and P. Blaha, "Lyapunov-function-based flux and speed observer for AC induction motor sensorless control and parameters estimation," IEEE Trans. Ind. Electron., vol. 53, no. 1, pp. 138-145, Feb. 2006.

[47] G. C. Verghese and S. R. Sanders, "Observers for flux estimation in induction machines," IEEE Trans. Ind. Electron., vol. 35, no. 1, pp. 85-94, Feb. 1988.

[48] Z. Zhang, H. Xu, L. Xu, and L. E. Heilman, "Sensorless direct fieldoriented control of three-phase induction motors based on 'sliding mode' for washing-machine drive applications," IEEE Trans. Ind. Appl., vol. 42, no. 3, pp. 694-701, May/Jun. 2006.

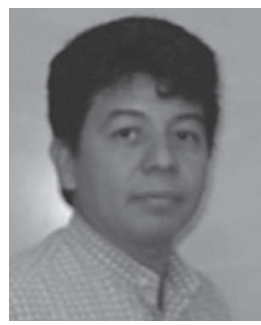

Bernardino Castillo-Toledo received the B.Sc. degree in electrical engineering from the National Polytechnic Institute (IPN), Mexico City, Mexico, the M.Sc. degree from the Research and Advanced Studies Center of the National Polytechnic Institute (CINVESTAV-IPN), Guadalajara, Mexico, and the Ph.D. degree from the University of Rome "La Sapienza," Rome, Italy, in 1981, 1985, and 1992, respectively.

He was a Lecturer with the School of Electrical and Mechanics Engineering, IPN, from 1985 to 1989 . From 1985 to 1995 , he was with the Automatic Control Section, Department of Electrical Engineering, CINVESTAV-IPN, and, since 1995, with CINVESTAV-IPN, Guadalajara Campus. He held several research stages with the University of Rome "La Sapienza" and University of L'Aquila, L'Aquila, Italy, and was a visiting Professor at the Laboratoire d'Automatique et d'Analyse des Systemes, French Council for Scientific Research, during 1999 to 2000, and at the University of Compiègne, Compiegne, France, during the first semester of 2002. His main research interests include nonlinear control design, the robust regulation problem, and the application of artificial neural networks and fuzzy logic techniques to control and fault diagnosis of dynamical systems.

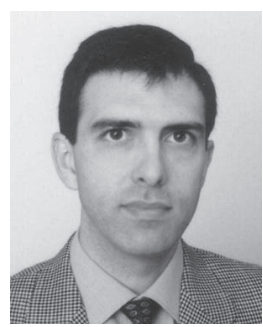

Stefano Di Gennaro received the M.S. degree (summa cum laude) in nuclear engineering and the $\mathrm{Ph} . \mathrm{D}$. degree in system engineering from the University of Rome "La Sapienza," Rome, Italy, in 1987 and 1992, respectively.

Since October 1990, he has been with the Department of Electrical and Information Engineering, University of L'Aquila, L'Aquila, Italy, where he was an Assistant Professor of Automatic Control and, since 2001, has been an Associate Professor of Automatic Control. He holds courses on automatic and nonlinear controls. He is also with the Center of Excellence DEWS-Design methodologies of Embedded controllers, Wireless interconnect and Systemson-chip, University of L'Aquila. In 1986, he was a Visiting Scientist at the Nuclear Research Center ENEA, Casaccia. He has been a Visiting Professor at the Laboratoire des Signaux et Systémes, Centre National de la Recherche Scientifique (CNRS), Paris, at the Department of Electrical Engineering, Princeton University, Princeton, NJ, at the Department of Electrical Engineering and Computer Science, University of California, Berkeley, and at the Advanced Studies and Research Center, National Polytechnic Institute, Unidad Ciudad de México and Unidad Guadalajara, México. He is working in the area of hybrid systems, regulation theory, and applications of nonlinear control. 


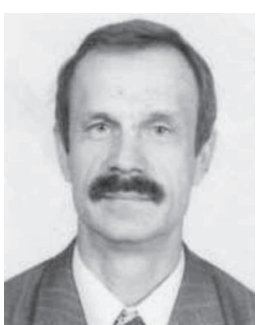

Alexander G. Loukianov was born in Moscow, Russia, in 1946. He received the B.S. degree from the Polytechnic Institute, Moscow, Russia, in 1975 and the $\mathrm{Ph} . \mathrm{D}$. degree in automatic control from the Institute of Control Sciences, Russian Academy of Sciences, Moscow, Russia, in 1985.

He was with the Institute of Control Sciences in 1978 and was the Head of the Discontinuous Control Systems Laboratory from 1994 to 1995 . From 1995 to 1997 , he held a visiting position at the University of East London, London, U.K., and since April 1997, he has been with the Research and Advanced Studies Center of the National Polytechnic Institute, Guadalajara Campus, Mexico, as a Professor of Electrical Engineering graduate programs. From 1992 to 1995, he was in charge of an industrial project between the institute and the largest Russian car plant and also several international projects supported by the International Association and Specific International Scientific Cooperation Activities-COPERNICUS, Brussels. He has published more than 100 technical papers in international journals, books, and conferences and has been a Reviewer for different international journals and conferences. His research interests include nonlinear system robust control and variable structure systems with sliding modes as applied to dynamical plants with delay, electric drives, and power systems control, robotics, spice, and automotive control.

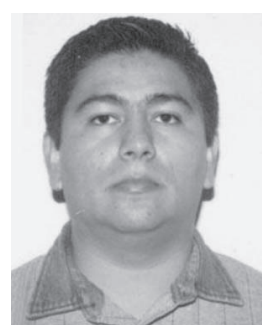

Jorge Rivera was born in El Rosario, Sinaloa, México, in 1975. He received the B.Sc. degree from the Technological Institute of the Sea, Mazatlán, México, in 1999, and the M.Sc. and Ph.D. degrees in electrical engineering from the Advanced Studies and Research Center, National Polytechnic Institute, Guadalajara, México, in 2001 and 2005, respectively

Since 2006, he has been with the Universidad de Guadalajara, Guadalajara, México, as a full-time Professor in the Centro Universitario de Ciencias Exactas e Ingenierías, Departamento de Electrónica. His research interests focus on regulator theory, sliding mode control, discretetime nonlinear control systems, and their applications to electrical machines. 